\title{
The internal structure of a vortex in a two-dimensional superfluid with long healing length and its implications
}

\author{
Avraham Klein $^{\mathrm{a}}$, Igor L. Aleiner ${ }^{\mathrm{b}}$, Oded Agam ${ }^{\mathrm{a}, \mathrm{b}}$ \\ ${ }^{a}$ The Racah Institute of Physics, The Hebrew University of Jerusalem, 91904, Israel \\ ${ }^{b}$ Physics Department, Columbia University, New York, NY 10027, USA
}

\begin{abstract}
We analyze the motion of quantum vortices in a two-dimensional spinless superfluid within Popov's hydrodynamic description. In the long healing length limit (where a large number of particles are inside the vortex core) the superfluid dynamics is determined by saddle points of Popov's action, which, in particular, allows for weak solutions of the Gross-Pitaevskii equation. We solve the resulting equations of motion for a vortex moving with respect to the superfluid and find the reconstruction of the vortex core to be a non-analytic function of the force applied on the vortex. This response produces an anomalously large dipole moment of the vortex and, as a result, the spectrum associated with the vortex motion exhibits narrow resonances lying within the phonon part of the spectrum, contrary to traditional view.
\end{abstract}

Keywords: quantum vortices; two dimensional superfluid; Popov's equations; Gross-Pitaevskii equations.

PACS: 67.10.-j (Quantum fluids: general properties)

67.10.Jn (Transport properties and hydrodynamics)

67.85.-d (Ultracold gases, trapped gases)

67.85.De (Dynamics properties of condensates; excitations, and superfluid flow)

\section{Introduction}

Hydrodynamics of superfluids, and in particular the dynamics of quantum vortices, have been studied extensively since the discovery of superfluidity in liquid Helium in the late 1930s [1]. However, even today certain aspects of superfluidity are not fully understood. For instance, the problems of the vortex mass and of the force acting on it are still controversial (see Ref. [2] and references therein), and there is no full theoretical understanding of the excitation spectrum of superfluids (for recent works on this subject see, e.g., $[3,4]$ ).

Interest in the theory of superfluidity has revived considerably in recent years, ever since atom cooling techniques opened up new experimental avenues for realization of superfluids using Bose-Einstein condensates (BEC) of cold atoms $[5,6,7]$. These systems provide precise tomography of the condensate structure, and are used nowadays to gather additional information on problems 
which were studied, in the past, using superfluid Helium (for a review see [8] and references therein). Examples of these are dynamics of vortices $[9,10,11,12,13]$, Kelvin waves [14], vortex lattices [15, 16], Tkachenko waves [17], vortex tangles [18], and quantum turbulence [19].

However, whereas liquid Helium represents a superfluid in the strong coupling regime, BECs are usually described by a weakly coupled theory, as the strength of interatomic interactions can be controlled and tuned to be very small. One difference between these two types of systems manifests itself in the size of the vortex core. In the strong coupling regime the vortex core is, effectively, of the interatomic distance, while in the weak coupling limit it is much larger. The core size of the vortex has, in turn, important implications for the vortex dynamics. For vanishingly small vortex cores, the Kelvin circulation theorem [20] implies that a vortex can only move together with its surrounding fluid, i.e. one cannot apply a force on a vortex. In the other extreme, i.e. when the vortex core is very large compared to the interatomic distance, forces acting on vortices are non-perturbative. They may deform the vortex core and play an important role in the dynamics of the vortex. In other words the hydrodynamical description of a superfluid with vortices should include not only the positions and vorticity charges of the vortices, but also (at the very least) their dipole moments.

Most studies of quantum vortices have employed the mean field description given by the Gross-Pitaevskii equation (GPE), which provides a good approximation in the weak coupling regime. However, the question concerning the internal structure of the vortex and, in particular, its description by weak solutions of the GPE has largely been overlooked. (By weak solutions we refer to the case were the GPE is satisfied everywhere in space except for a set of zero measure).

In this paper we show that these weak solutions necessarily appear when a force is applied to a vortex. Moreover, they manifest themselves in an anomalously large dipole moment. We construct the hydrodynamical description of a spinless superfluid in two dimensions, taking into account the dipole moment of the vortices. This description applies over distances larger than the vortex core and when vortices are far apart. A prominent prediction of this effective theory is the existence of low-energy excitation-levels of vortex core. We show that these energy level are long-lived, and characterize their influence on the elastic scattering of phonons from vortices.

The rest of the paper is organized as follows. In the following section we present a qualitative discussion of our result. In Sec. 3, we review Popov's field theory [21] of superfluids where vortices are introduced as constraints. Then, in Sec. 4, we present our solution of the Popov equations for the case of a pinned vortex in a uniform flow. We first show the numerical solution of the problem and then provide analytical understanding by solving the Bogolyubov equations and matching the solution to the weak solution of the GPE in close vicinity to the vortex core. Next, in Secs. 5 and 6 , we construct the effective field theory which takes into account the dipole-moment of the vortex, derive the corresponding equations of motion, and solve them in order to describe the classical oscillatory motion of a vortex and its coupling to phonons. In Sec. 7 
we quantize the vortex motion. Finally, in Sec. 8, we conclude and discuss some future directions of the study of the non-analytic core deformation of vortices.

\section{Qualitative discussion and summary of the results.}

The hydrodynamic equations describing stationary solutions of a superfluid far from vortex cores are the conservation of mass,

$$
\vec{\nabla} \cdot(n \vec{v})=0,
$$

where $n$ and $\vec{v}$ are the superfluid density and velocity respectively, and the requirement of potential flow outside the vortex,

$$
\vec{\nabla} \times \vec{v}=0 .
$$

Finally, for potential flow and zero-entropy evolution, the Bernoulli equation (in the local approximation for the equation of state) reads,

$$
\vec{\nabla}\left(\frac{\vec{v}^{2}}{2}+n\right)=0 .
$$

Here, and from this point onwards, we work in dimensionless units such that density is measured in units of the equilibrium density, $n_{0}$, the length is measured in units of the healing length $\xi=1 / \sqrt{\lambda n_{0}}$ where $\lambda$ is the dimensionless coupling constant, and the velocity, $\vec{v}$, is measured in units of $\hbar /(m \xi)$ where $m$ is the particle's mass. Accordingly, the frequency is measured in units of healing frequency $\hbar /\left(m \xi^{2}\right)$, and the energy is measured in units of $\hbar^{2} /\left(m \xi^{2}\right)$. The speed of sound in our units is one. The weak coupling limit, on which we focus, is realized when $1 / \lambda=n_{0} \xi^{2} \gg 1$, namely when the number of particles within a square of size of the healing length squared is much larger than one.

For the sake of convenience we shall also represent vectors in the complex plane, e.g. $v=v_{x}+i v_{y}$. In this representation the solution of a single vortex, with unit vorticity, located at the origin is given by $v=i / z^{*}$, where $z=x+i y$ is the complex coordinate and $(\cdot)^{*}$ denotes complex conjugation. From Eq. (1c) it thus follows that the density is

$$
n=1-1 /\left(2|z|^{2}\right)
$$

sufficiently far from the vortex core, i.e. for $|z| \gg 1$ (at short distances the gradient terms in the equation of state become important and will be discussed later).

Consider, now, a single vortex moving with constant velocity $-\vec{v}_{c}$, and let us choose a moving coordinate frame attached to the vortex core (it corresponds to a fixed vortex and a superfluid flow $\vec{v}_{c}$ far from the vortex). If one could ignore Eq. (1c) and require $n=1$ (i.e. an incompressible liquid model), the

solution of the first two equations of Eqs. (1a-1b) would be a superposition of the two contributions to the superfluid velocity: $v \equiv v_{x}+i v_{y}=i / z^{*}+v_{c}$, or in 
other words, the vortex moves with the flow. However, the nonlinear coupling between the velocity and density (1c), modifies the solution such that, to linear order in $v_{c}$, one finds:

$$
v \simeq v_{c}+\frac{i}{z^{*}}+\frac{i d^{*}(|z|)}{\left(z^{*}\right)^{2}}+\mathcal{O}\left(\frac{v_{c}}{|z|^{2}}\right), \quad|z| \gg 1,
$$

where $d=d_{x}+i d_{y}$ is the dipole moment describing the deformation of the vortex-density shape under the effect of the external flow,

$$
d=-i v_{c} \ln |z|+d(1)
$$

Equations $(3-4)$ show that the virtual position of the vortex core, when viewed from far distances, is shifted with respect to its actual position by $d$. This shift is proportional to the velocity of the vortex and grows logarithmically with the distance. The same shift is also manifested in the superfluid density around the vortex.

One of the main conclusions of the present paper concerns the dependence of the shift $d(1)$ on the superfluid velocity $v_{c}$. Naively, one could estimate $d(1) \simeq-i \beta v_{c}$, where $\beta$ is a constant of the order of unity, so it does not have any visible effect on the background of the logarithmic function. However, contrary to this naive view, we show that the dipole moment has a non-trivial and non-analytic dependence on the velocity,

$$
d(1)=-\frac{i v_{c}}{\alpha^{2}} \ln \frac{1}{\left|v_{c}\right|},
$$

where $\alpha=0.8204 \ldots$ is a constant that describes the density profile near a stationary vortex $\left(v_{c}=0\right)$,

$$
n=\alpha^{2}|z|^{2}+\ldots, \quad|z| \ll 1 .
$$

The origin of the logarithmic dependence of dipole moment (5) is in a so called weak solution of the stationary Gross-Pitaevskii equation which is obtained from the variation of the functional:

$$
E\left[\psi, \psi^{*}\right]=\int d^{2} r\left[\frac{|\nabla \psi|^{2}}{2}+\frac{\left(|\psi|^{2}-1\right)^{2}}{2}\right] .
$$

Numerical solutions of the corresponding stationary equations (obtained with the help of Popov's formalism which will be described in Sec. 3) demonstrate that the logarithmic shift of the virtual position of the vortex indeed emerges from the singular structure of the vortex core. Figures 1 shows the plots for density profile. The solid line in Figs. 1 b,c) is the following analytic solution for distances much smaller than the healing length

$$
\psi=\frac{i v_{c}}{2 \alpha}\left[Z-\frac{1}{Z^{*}}+\ln \left(Z Z^{*}\right)\right],
$$



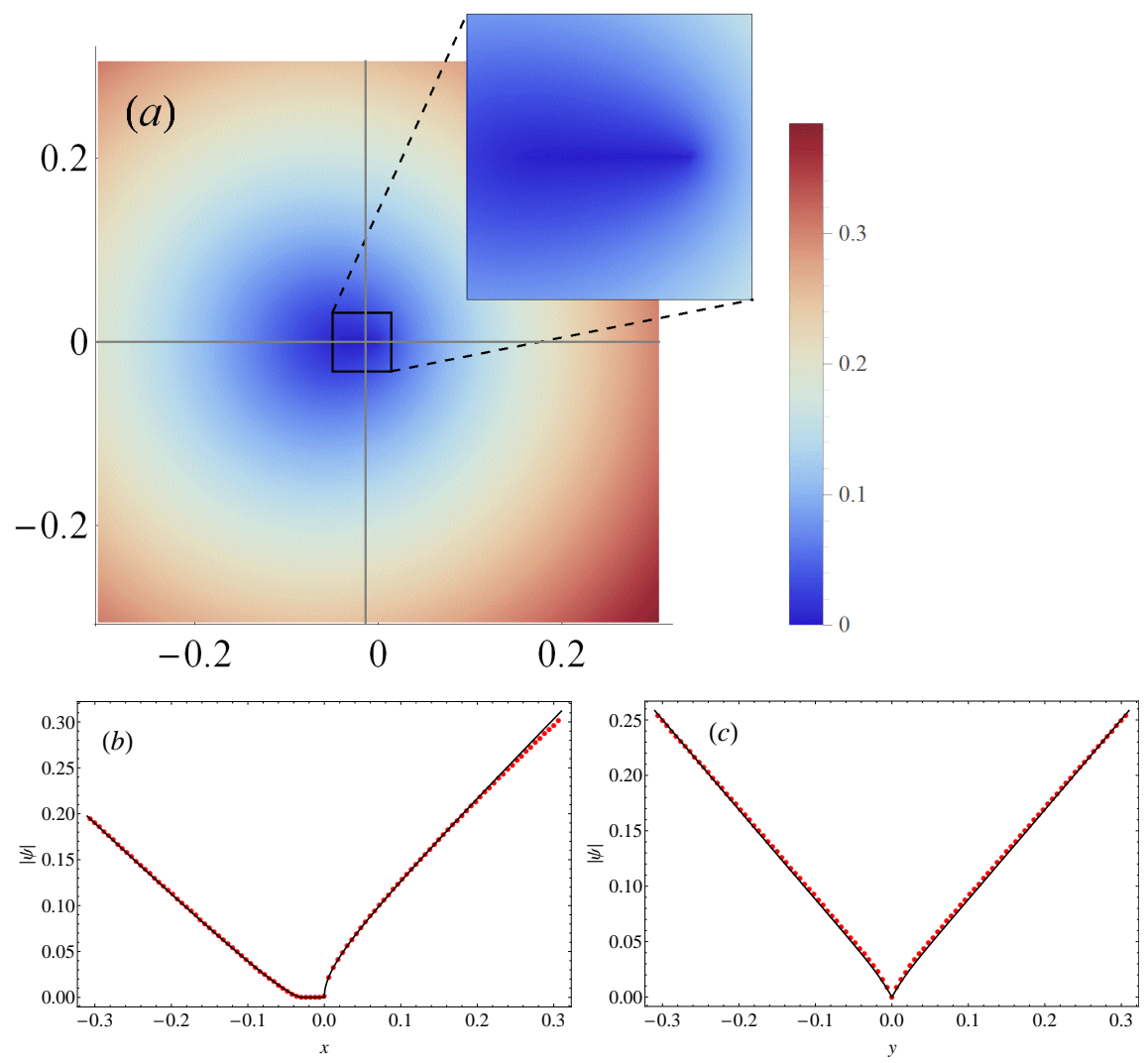

Figure 1: The square root of the density, $|\psi|$, of a superfluid near the core of a moving vortex (either vortex moves in positive $y$-direction or the supercurrent is along negative $y$-direction for a vortex in rest) : (a) Two dimensional density plot. (b) A section of $|\psi|$ along the horizontal gray line in upper panel. (c) A section of $|\psi|$ along the vertical gray line of the upper panel which bisect the cut at its midpoint. The red dots are the result of our numerical solution while the solid line is obtained from formula (8).

where

$$
Z=\frac{\alpha^{2} z}{i v_{c}}+\sqrt{\left(\frac{\alpha^{2} z}{i v_{c}}\right)^{2}-1}, \quad \text { and } \quad|z| \ll 1 .
$$

(The function $\sqrt{\zeta^{2}-1}$ is defined to be analytic in the entire complex plane except for a cut along the real axis between the points $\zeta=-1$ and $\zeta=1$.) This solution is obtained by approximating the Gross-Pitaevskii equation with the Laplace equation, see Sec. 4. From here it follows that the solution of a moving vortex exhibits a non-analytic behavior in a form of a cut of length proportional to the velocity $v_{c}$. One branch point of the cut is located at the point in which the vortex is pinned, and the cut extends in a direction perpendicular to the velocity, $v_{c}$, i.e. in the same direction as the Magnus force acting on the vortex.

This nonanalytic solution of the Gross-Pitaevskii equation should be under- 
stood as a weak solution. Namely, in similar manner to shock waves in hydrodynamics, it represents a saddle point of the energy (7) but it is not an analytic solution of the saddle point's equations. The weak solution is valid only outside some limited region of measure zero (the vicinity of the cut in our case). Within that region the description of the superfluid by the functional (7) does not hold, and additional physics should be taken into account by proper regularization. Nevertheless, the weak solution implies that, contrary to the traditional view, a vortex in the weak coupling limit possesses an anomalous contribution to a dipole-like degree of freedom which is associated with the length and the direction of the cut. The physical results which we will obtain from this property do not depend on the regularization scheme at all.

The appearance of the anomalous dipole moment (5) has a profound effect on the low-energy dynamics of the vortices. We shall enumerate these effects here and relegate their detailed discussion to the corresponding sections:

1. The dipole moment leads to similar non-analytic dependence of the added mass (see, e.g., Ref. [22]) of the vortex, $M_{v} \simeq m\left(n_{0} \xi^{2}\right) \ln (1 /|v|)$ where $|v|$ is the velocity of the vortex with respect to the superfluid, see Sec. 5.2 for more details.

2. Under the effect of the Magnus force, the vortex exhibits a periodic circular motion with frequency $\Omega \propto \frac{\hbar n_{0}}{M_{v}} \simeq \frac{\hbar}{m \xi^{2} \ln (1 \| v \mid)} \ll \frac{\hbar}{m \xi^{2}}$. Thus, vortex oscillations with small amplitude have frequencies within the phonon spectrum, contrary to the widely accepted view in the literature, see Sec. 6.2.

3. The circulating vortex emits sound waves (phonons) but this emission is suppressed by an additional factor of $1 /[\ln (1 /|v|)]^{3}$, see Sec. 6.3.2. Therefore, the excited states of the vortices are well defined resonances.

4. Quantization of the periodic motion of the vortex, leads to quantization of the radius of the circular motion which determines, in turn, the discrete energies of those resonances. These resonances lie within the phonon spectrum, provided that the superfluid has a long healing length, $n_{0} \xi^{2} \gg 1$, see Sec. 7 .

\section{Popov's formalism.}

In this section we briefly review the derivation of Popov's representation of the dynamics of a two-dimensional compressible superfluid [21].

To shorten the notation, we represent vectors in the two dimensional space using arrow, e.g. $\vec{r}=(x, y)$, or $\vec{v}=\left(v_{x}, v_{y}\right)$. We also use 3 -vectors notation in which the first component is time-like and the two other are space-like. These will be represented by boldface letters, e.g. $\mathbf{x}=(t, x, y)$, and $\boldsymbol{\partial}=\left(\partial_{t}, \partial_{x}, \partial_{y}\right)$.

All zero-temperature quantum properties of the superfluid can be obtained by a suitable differentiation of the "partition function"

$$
\mathcal{Z}=\int \mathcal{D} \psi^{*} \mathcal{D} \psi \exp \left(\frac{i \mathcal{S}}{\lambda}\right)
$$


with the action

$$
\mathcal{S}=\int d^{3} x i \psi^{*} \partial_{t} \psi-\int d t E\left[\psi, \psi^{*}\right],
$$

and the energy functional $E\left[\psi, \psi^{*}\right]$ given by Eq. (7). The interaction constant $\lambda \ll 1$, therefore, plays the role of the Planck constant which allows for the semiclassical treatment of the problem in the weak coupling regime.

\subsection{Derivation of Popov's action.}

The derivation of Popov's theory of superfluids is comprised of two mains steps. The first is the Madelung transformation:

$$
\psi=\sqrt{n} \exp (i \theta)
$$

where $n$ is the density of the superfluid and $\theta$ is its phase, which transforms the action (10) to

$$
\mathcal{S}=-\int d^{3} x\left[v_{0} n+\frac{n|\vec{v}|^{2}}{2}+\frac{|\vec{\nabla} n|^{2}}{8 n}+\frac{(n-1)^{2}}{2}\right] .
$$

where $\mathbf{v}=\left(v_{0}, v_{x}, v_{y}\right)$ are given by

$$
\mathbf{v}=\partial \theta
$$

The well-known disadvantage of this representation of (11) is that $\theta$ is a multi-valued function of the coordinate, and therefore taking into account configurations with moving vortices is somewhat inconvenient. The second step of the derivation introduces new single-valued fields and vortices as a way to resolve Eq. (13) as well as the multi-valuedness of the field $\theta$.

We consider, instead of $\theta$, a three-component field of the velocity, $\mathbf{v}(\mathbf{x})$. According to Eq. (13) these components are not independent,

$$
\epsilon_{i j k} \partial_{j} v_{k}=2 \pi J_{i}^{V}
$$

where $\epsilon_{i j k}$ is the unit antisymmetric tensor. In the right hand side of Eq. (14) are the components of the 3 -vector representing the vortex current. These take into account that $\theta$ is not a single-valued function and can be changed by the multiples of $2 \pi$ while traveling along any contour encompassing a vortex line $R_{l}(t)$ :

$$
J_{0}^{V}=\sum_{l} \sigma_{l} \delta\left[\vec{r}-\vec{R}_{l}(t)\right] ; \quad \vec{J}^{V}=\sum_{l} \sigma_{l} \partial_{t} \vec{R}_{l} \delta\left[\vec{r}-\vec{R}_{l}(t)\right] .
$$

Here, $\vec{R}_{l}(t)$ and $\sigma_{l}$ denote the position and the vortex charge of the $l$-th vortex. The vortex current is analogous to the particle current in the usual electrodynamics and, by construction, satisfies the continuity equation

$$
\partial \cdot \boldsymbol{J}^{V}=0 .
$$


Introducing gauge fields $\boldsymbol{A}=\left(a_{0},-a_{x},-a_{y}\right)$ to resolve constraint (14), we rewrite Eq. (9) as

$$
\mathcal{Z}=\left[\prod_{l} \int \mathcal{D} \boldsymbol{R}_{l}(t)\right] \int \mathcal{D} n \mathcal{D} \mathbf{v} \mathcal{D} \boldsymbol{A} \exp \left[\frac{i \mathcal{S}}{\lambda}+\frac{i}{\lambda} \int d^{3} x A_{i}\left(\epsilon_{i j k} \partial_{j} v_{k}-2 \pi J_{i}^{V}\right)\right],
$$

where action $\mathcal{S}$ is given by Eq. (12).

The integration over all three components of the velocity $\mathbf{v}$ in Eq. (17) can be performed exactly, leading to Popov's non-linear electrodynamics:

$$
\mathcal{Z}=\left[\prod_{l} \int \mathcal{D} \boldsymbol{R}_{l}(t)\right] \int \mathcal{D} \boldsymbol{A} \exp \left[\frac{i \mathcal{S}_{P}}{\lambda}\right],
$$

where the vortex current is defined in Eq. (15) and Popov's action is given by

$$
\mathcal{S}_{P}=\int d^{3} x\left[\frac{\vec{E}^{2}}{2 B}-\left(\frac{|\vec{\nabla} B|^{2}}{8 B}+\frac{(B-1)^{2}}{2}\right)-2 \pi \boldsymbol{J}^{V} \cdot \boldsymbol{A}\right] .
$$

Popov's field $\vec{E}=\left(E_{x}, E_{y}\right)$ and $B$ are analogous to electric and magnetic fields in two-dimensional electrodynamics

$$
B=\vec{\nabla} \times \vec{a} ; \quad \vec{E}=-\partial_{t} \vec{a}-\vec{\nabla} a_{0} .
$$

The last term of the action (19) has the form of the interaction of the particles (vortices) with the fields. The gauge invariance of the theory (18) is guarded by the vortex current conservation (16).

The physical superfluid current $\vec{j}$ and density $n$ can expressed in terms of Popov's fields as

$$
\left(n, j_{x}, j_{y}\right)=\left(B, E_{y},-E_{x}\right)
$$

\subsection{Popov's equations}

Let us now write down the equations of motion which follow from Popov's formalism. The first dynamical equation, analogous to Faraday's law, is obtained directly from the relation (20) of the fields, $\vec{E}$ and $B$, to the gauge fields, A:

$$
\partial_{t} B=-\vec{\nabla} \times \vec{E}
$$

From comparison of Eq. (22a) with Eq. (21) it follows that this equation is just the requirement of conservation of physical mass charge $\partial_{t} n+\vec{\nabla} \cdot \vec{j}=0$.

The other Maxwell equations are obtained by varying of the action (19) with respect to the vector-potential $\boldsymbol{A}$. Variation with respect to $a_{0}$ gives an analogue of Gauss' law:

$$
\vec{\nabla} \cdot\left(\frac{\vec{E}}{B}\right)=2 \pi J_{0}^{V}(\mathbf{r})
$$

where vortex density and currents are defined in Eq. (15). This equation re-

places the quantization condition of the velocity circulation. The third equation, 
analogous to Amper's law, is obtained by variation of the action with respect to $\vec{a}$ :

$$
\hat{\varepsilon} \vec{\nabla}\left[\frac{\vec{E}^{2}}{2 B^{2}}+B-\frac{1}{2 \sqrt{B}} \vec{\nabla}^{2} \sqrt{B}\right]=\vec{J}^{V}(\mathbf{r}, t)+\partial_{t}\left(\frac{\vec{E}}{B}\right),
$$

where $\hat{\varepsilon}$ is an antisymmetric tensor of the second rank acting on the spatial coordinates.

A further advantage of the action (19) is that it allows one to immediately find the force acting on a vortex. Varying the action with respect to the vortex position, we find

$$
\sum_{l} \int d t \vec{F}_{l} \delta \vec{R}_{l}=-\delta\left(2 \pi \int d^{3} \mathbf{x} \boldsymbol{J}^{V} \cdot \boldsymbol{A}\right) .
$$

Direct calculation utilizing Eqs. (20) gives

$$
\vec{F}_{l}=2 \pi \sigma_{l} \vec{f}_{l} ; \quad \overrightarrow{f_{l}} \equiv \vec{E}\left(\vec{R}_{l}, t\right)+B\left(\vec{R}_{l}, t\right) \hat{\varepsilon} \partial_{t} \vec{R}_{l}
$$

which is equivalent to the usual expression in linear electrodynamics.

Equation (23) is just an expression for the Magnus force provided that $\vec{E}$ and $B$ are understood as external fields which do not include the fields created by the vortex itself. In standard electrodynamics such exclusion of the selfinteraction is easily performed because the equations of motion for the fields are linear and the superposition principle holds. Here, Eqs. (22a-22b) are nonlinear and exclusion of the self-interaction is not a trivial task. We postpone our consideration of this question until we have had a chance to study in detail the fine structure of the core of a moving vortex (in Sec. 4), and to obtain the equation of motion for the vortex (in Sec. 5).

\subsection{Galilean invariance}

Popov's action and equations of motion preserve the Galilean invariance of the original model. One can show that the transformations

$$
\begin{aligned}
& a_{0}(t, \vec{r}) \rightarrow a_{0}(t, \vec{r}-\vec{v} t)+\vec{v} \cdot \vec{a}(t, \vec{r}-\vec{v} t) ; \quad \vec{a}(t, \vec{r}) \rightarrow \vec{a}(t, \vec{r}-\vec{v} t) ; \\
& \vec{B}(t, \vec{r}) \rightarrow \vec{B}(t, \vec{r}-\vec{v} t) ; \quad \vec{E}(t, \vec{r}) \rightarrow \vec{E}(t, \vec{r}-\vec{v} t)-B(t, \vec{r}-\vec{v} t) \hat{\varepsilon} \vec{v} ; \\
& \vec{R}_{i}(t) \rightarrow \vec{R}_{i}(t)+\vec{v} ; \\
& J_{0}^{V}(t, \vec{r}) \rightarrow J_{0}^{V}(t, \vec{r}-\vec{v} t) ; \quad \vec{J}^{V}(t, \vec{r}) \rightarrow \vec{J}^{V}(t, \vec{r}-\vec{v} t)+\vec{v} J_{0}^{V}(t, \vec{r}-\vec{v} t) ;
\end{aligned}
$$

(with $\vec{v}$ being any constant velocity) leave equations of motion (22) and the force acting on a vortex (23) intact. Under the same transformation, Popov's action acquires an extra term

$$
\delta \mathcal{S}_{P}=\int d^{3} x\left[\frac{\vec{v}^{2} B}{2}-\vec{E} \hat{\varepsilon} \vec{v}\right],
$$

which is just the change of the total kinetic energy in the moving frame. As each of the terms is a total derivative, they do not influence the dynamics of the system. 


\subsection{Relation to the Gross-Pitaevskii equation.}

Everywhere in space, where there are no vortices and $B>0$, it is possible to express the solution of Eqs. (22) in terms of the Gross-Pitaevskii equation

$$
i \partial_{t} \Psi=-\frac{1}{2} \vec{\nabla}^{2} \Psi+\left(|\Psi|^{2}-1\right) \Psi .
$$

Indeed, substituting

$$
B=|\Psi|^{2} ; \quad \vec{E}=\frac{i}{2} \hat{\varepsilon}\left[\Psi^{*} \vec{\nabla} \Psi-\Psi \vec{\nabla} \Psi^{*}\right],
$$

into Eqs. (22), we find those equation are satisfied wherever the vortex density and current equal zero. The advantage of Popov's variables is that they allow us to include configurations where equation (26) is not satisfied on a set of measure zero, or in other words weak solutions of the GPE. An example of this situation is a pinned vortex in a superfluid flow, which we describe in the next section.

\section{Solution of the Popov equations for a pinned vortex in a superfluid flow}

In this section we discuss the solution of Popov's equations for a pinned vortex in an otherwise constant superfluid flow. We first present our numerical procedure for the solution of this problem, and show that it is, in fact, a weak solution which exhibits a cut singularity within the vortex core. Next we provide an analytic interpretation of the result. To this end we first solve the Bogolyubov equations which provide the perturbative solution of the problem far from the cut-singularity. Then we solve the Gross-Pitaevskii equation deep within the vortex core by matching the solution to the perturbative result.

As we are interested in a stationary solution of Eqs. (22), it may be described by two real fields, $\rho=\sqrt{B}$ and $a_{0}$ (the electric field is given by $\vec{E}=-\vec{\nabla} a_{0}$ ), thus Popov's equations reduce to the following pair of equations:

$$
\begin{aligned}
-\vec{\nabla} \cdot \frac{\vec{\nabla} a_{0}}{\rho^{2}} & =2 \pi \sigma \delta\left(\vec{r}-\vec{R}_{0}\right) \\
\hat{H} \rho & =0
\end{aligned}
$$

where $\hat{H}$ is a nonlinear operator which is defined as

$$
\hat{H} \rho=\left(\frac{1}{2} \vec{\nabla}^{2}-\frac{1}{2}\left|\frac{\vec{\nabla} a_{0}}{\rho^{2}}\right|^{2}+1-\rho^{2}\right) \rho .
$$

The boundary conditions on $\vec{E}$ are that far from the vortex the superfluid current is constant, namely $\vec{E}(r \rightarrow \infty)=-\hat{\varepsilon} \mathbf{v}_{c}$. 


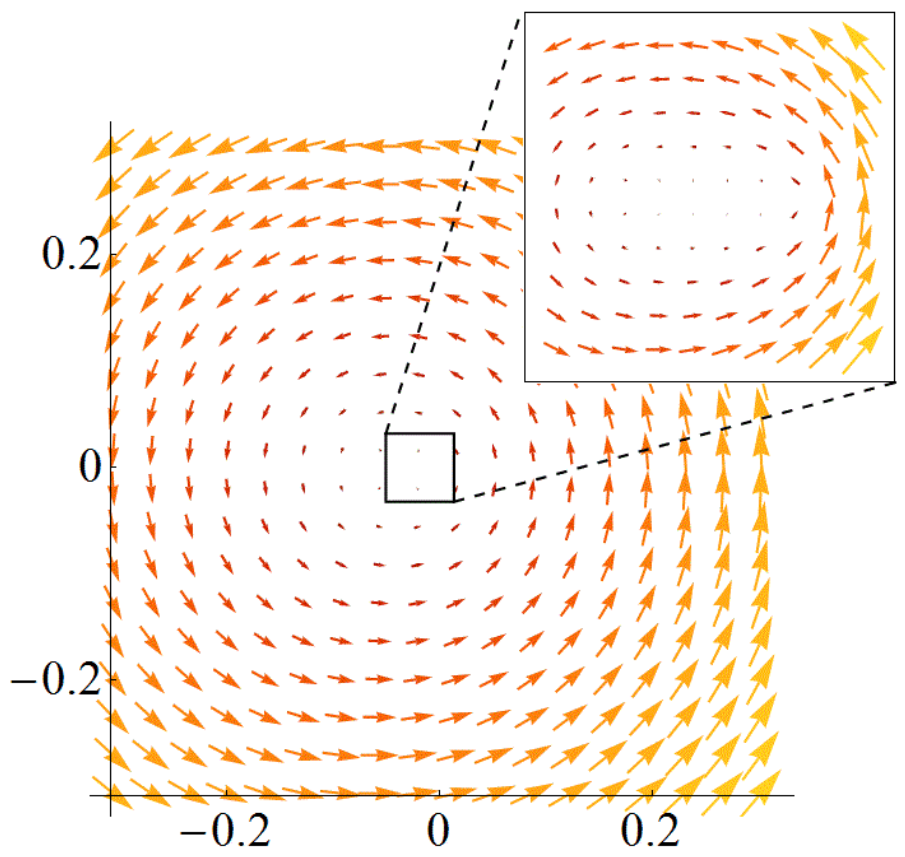

Figure 2: The superfluid current in the vortex core of a pinned vortex in a uniform flow, $\vec{j}=\hat{\epsilon} \vec{E}$. The inset shows a magnified view of the region near the cut similar to Fig. 1 .

\subsection{Numerical solution}

The numerical results for the solution of the above equations for the square root of the density and the superfluid current in the vicinity of the vortex core are depicted in Fig. 1, and Fig. 4, respectively. Here we sketch the numerical procedure used in order to obtain these results. Additional details can be found in Appendix A.

Working with two real fields, $a_{0}$ and $\rho$, simplifies the numerical task. For the numerical procedure it is efficient to apply the external superfluid flow by placing the vortex in a very large square box and slightly shifting its position from the center of the box. The boundary conditions on the box are equivalent to the creation of "image" vortices and anti-vortices. The shift of the vortex from the center results in a superfluid flow at the position of the vortex, as illustrated in Fig. 3. To see how this works, consider the boundary conditions of a superfluid in a box. The component of the superfluid current normal to the boundaries must be zero, and therefore the electric field must be normal to the boundaries. Thus we have the electrostatic problem of a grounded metal box, with Dirichlet boundary conditions:

$$
a_{0}(x, \pm L / 2)=a_{0}( \pm L / 2, y)=0 .
$$

where $L$ is the size of the box. In analogy with the electrostatic problem, we can remove the boundaries by creating a lattice of image "charges", i.e. vortices with alternating vorticities. The superfluid density is the same for a vortex and antivortex, thus we need periodic boundary conditions for $\rho$. Since $\rho \geq 0$, we chose to use the equivalent and slightly more convenient Neumann boundary 


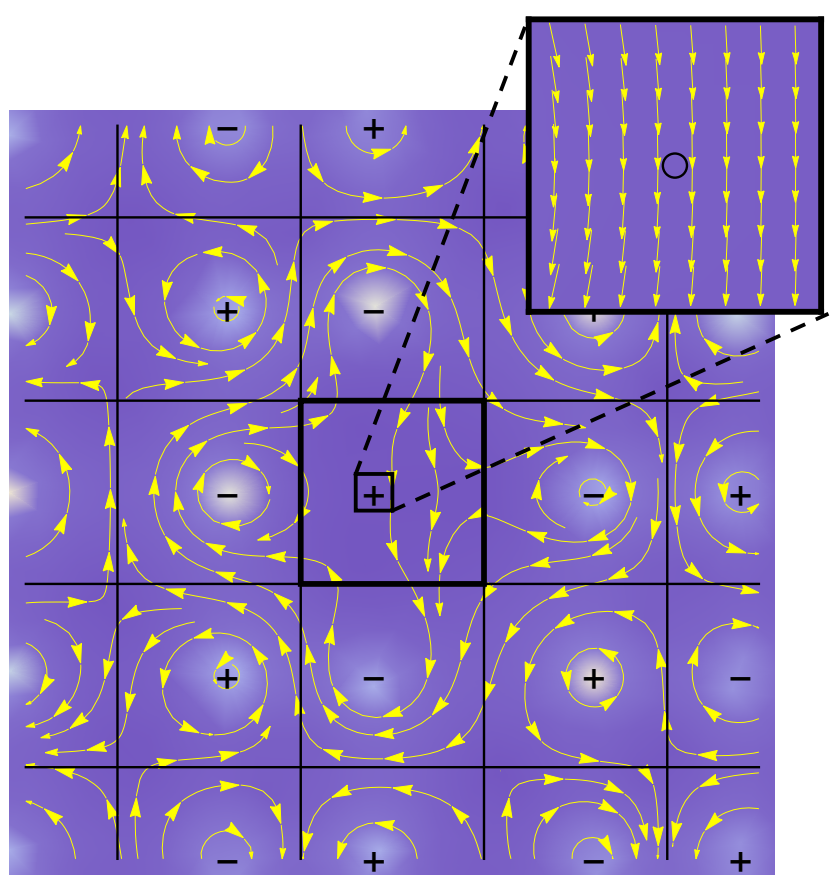

Figure 3: The lattice of image vortices generated due to Dirichlet boundary conditions, and the effective current (total current minus the current created by the central the vortex) produced when the vortex is shifted from the central point of the domain. The inset shows a magnified view of the region of the vortex. Here, the circle represents the size of a vortex, i.e. a circular region with radius equals to the healing length. This size is much smaller that the scale over which the flow changes.

conditions

$$
\left.\hat{n} \cdot \vec{\nabla} \rho\right|_{\vec{r} \in \text { boundary }}=0,
$$

where $\hat{n}$ is the normal to the boundary. Shifting the vortex core from the center of the box, say by choosing $\vec{R}_{0}=(-\Delta x, 0)$, shifts and deforms the entire lattice and imposes a flow at $\vec{R}_{0}$, see Fig. 3 . This flow can be calculated to excellent numerical accuracy by solving the analogous electrostatic problem.

Equations such as Eqs. (28) can be solved via relaxation to equilibrium of the fictitious time-dependent problem:

$$
\hat{H} \rho=\frac{\partial \rho}{\partial t}
$$

The dynamical system of Eqs. (28a)-(30) has a convenient fluid-dynamical interpretation. Essentially, the equations describe diffusive dynamics of an interacting fluid. The fluid is vorticity-free except at a single point $\vec{R}_{0}$.

We solve the equations by discretizing the space and the time, iterating Eq. (30) and enforcing Eq. (28a) explicitly at the end of each time step, i.e. solving the resulting linear equation for $a_{0}$ for the temporal value of $\rho$. This procedure is similar that used in hydrodynamic problems (see e.g. Ref. [23] for an overview of this topic). 
Special consideration must be given to the discrete form of Eq. (28a), which describes a conservation law. Its discrete form should enforce the discrete version of the law, or in other words, the discrete form of the current conservation $\vec{\nabla} \times E=0$ (which follows from Eq. (22a) for stationary case). A convenient way to achieve this, when the dynamics are given by an action, is to to derive the equation by variation of the discretized action [24]. A full description of the algorithm may be found in Appendix A.

Finally, to deal with the singular behavior that emerges in the vicinity of the vortex location, $\vec{R}_{0}$, one needs fine discretization near the vortex, usually going to lattice constants of order $\Delta \sim 0.01-0.001$. On the other hand one should also maintain large system sizes, usually $L \sim 50-100$, which implies an excessively large number of lattice points. To resolve this problem we used multiple-resolution grids, often using 3 grids so that near the core singularity we had very high resolution, sometimes as high as $\Delta=0.0005$. We interpolated the grids to each other using bicubic splines.

There is no general method of insuring stability and correctness for nonlinear problems with singularities. Instead, we monitored the progress of the iteration and checked that the solution converged properly. In order to increase accuracy, we made sure that a high-resolution grid always surrounded $R_{v}$ in order to reduce the so called "infection region" of the singularity.

\subsection{Analytical interpretation}

The behavior of the density and the current obtained from the numerical solution is described by the analytic formula (8) (see fits in panels (b) and (c) of Fig. 1). Namely, the solution exhibits a cut singularity, indicative of weak solutions. The purpose of this section is to motivate this result.

\subsubsection{Bogolyubov equations and the necessity for weak solutions.}

Here we present the perturbative solution of a pinned vortex in an otherwise uniform flow obtained from the zero-mode solution of the Bogolyubov equations. For this purpose it will be convenient to represent spatial position using polar coordinates $r, \phi$.

We consider a single vortex with $\sigma=1$ fixed at $r=0$ and set the current to be fixed at large distances, i.e. $\vec{E}(r \rightarrow \infty)=-\hat{\varepsilon} \mathbf{v}_{c}$. Beyond the vortex core, representation (27) is valid. Moreover, at the distances $v_{c} \ll r \ll 1 / v_{c}$ the perturbation of the vortex density and current profile is small and we can look for a solution in the form

$$
\Psi(r, \phi)=e^{i \phi} \sqrt{n(r)}+U(r)+V^{*}(r) e^{i 2 \phi},
$$

The expected behavior of the solution at distances larger than the healing length,

$$
\Psi(r, \phi)=e^{i \phi+i \vec{v}_{c} \cdot \vec{r}} \sqrt{n(r)} \simeq e^{i \phi}+\frac{i v_{c} r}{2}+\frac{i v_{c}^{*} r}{2} e^{i 2 \phi}, \quad v_{c}=v_{c}^{x}+i v_{c}^{y},
$$

establishes the condition on amplitudes at $1 \ll r \ll 1 /\left|v_{c}\right|$ :

$$
U^{(2)}(r)=-V^{(2)}(r)=i v_{c} r
$$


where the meaning of the superscript (2) will become clear later on. Substituting Eq. (31) into time independent Eq. (26) and linearizing with respect to $U$ and $V$, we obtain the zero-mode of the Bogolyubov equations

$$
\begin{aligned}
& {\left[-\frac{1}{2}\left(\frac{d^{2}}{d r^{2}}+\frac{d}{r d r}\right)+2 n(r)-1\right] U(r)+n(r) V(r)=0} \\
& {\left[-\frac{1}{2}\left(\frac{d^{2}}{d r^{2}}+\frac{d}{r d r}\right)+\frac{2}{r^{2}}+2 n(r)-1\right] V(r)+n(r) U(r)=0 .}
\end{aligned}
$$

A convenient way to rewrite Eq. (33) is to introduce even and odd combinations $W_{ \pm}=U \pm V$ for which we obtain

$$
\begin{aligned}
& {\left[-\frac{1}{2}\left(\frac{d^{2}}{d r^{2}}+\frac{d}{r d r}\right)+3 n(r)-1+\frac{1}{r^{2}}\right] W_{+}(r)-\frac{1}{r^{2}} W_{-}(r)=0} \\
& {\left[-\frac{1}{2}\left(\frac{d^{2}}{d r^{2}}+\frac{d}{r d r}\right)+n(r)-1+\frac{1}{r^{2}}\right] W_{-}(r)-\frac{1}{r^{2}} W_{+}(r)=0 .}
\end{aligned}
$$

This form of the equations is useful for analysis of the solutions at large distances, $r \gg 1$.

Equations (33) form a coupled pair of second order differential equations and therefore have four independent solutions. One solution can be ruled out as it diverges exponentially at large distances: $U(r)=V(r)=\exp (2 r)$. Another solution can be also ruled out since it contains a component which is too rapidly divergent at short distances: $V(r) \simeq 1 / r^{2}$. The two remaining two solutions of Eq. (33) are connected by the invariance of the Wronskian:

$$
\frac{d}{d r} r\left\{U^{(2)} \frac{d U^{(1)}}{d r}+V^{(2)} \frac{d V^{(1)}}{d r}-[(1) \leftrightarrow(2)]\right\}=0 .
$$

One (exact) solution of Eq. (33) is easy to find

$$
\left(\begin{array}{l}
U^{(1)} \\
V^{(1)}
\end{array}\right)=\left(\begin{array}{l}
\frac{d \sqrt{n}}{d r}+\frac{\sqrt{n}}{r} \\
\frac{d \sqrt{n}}{d r}-\frac{\sqrt{n}}{r}
\end{array}\right)=\left\{\begin{array}{cc}
\left(\begin{array}{c}
1 / r \\
-1 / r
\end{array}\right)+\mathcal{O}\left(\frac{1}{r^{3}}\right), & r \gg 1 ; \\
\left(\begin{array}{c}
2 \alpha \\
\mathcal{O}\left(r^{2}\right)
\end{array}\right), & r \ll 1 .
\end{array}\right.
$$

This solution can be understood as a small translation of the vortex as a whole, but it does not satisfy the boundary condition of supercurrent flow at large distances (32).

The second solution of Eq. (33), which can be obtained using the above solution and the invariance of the Wronskian (35), does satisfy boundary conditions (32) [the solution at $r \gg 1$ is obtained from Eq. (34) with $W_{+}=$ $\left.W_{-} /\left(2 r^{2}\right)+\ldots, \mathbf{n}(r)=1-1 /\left(2 r^{2}\right)-1 /\left(2 r^{4}\right)-\ldots\right]$ :

$$
\left(\begin{array}{l}
U^{(2)} \\
V^{(2)}
\end{array}\right)=\frac{i v_{c}}{2}\left\{\begin{array}{cc}
\left(\begin{array}{cc}
r+\frac{\ln r}{r}+\frac{1}{2 r} \\
-r-\frac{\ln r}{r}+\frac{1}{2 r}
\end{array}\right)+\mathcal{O}\left(\frac{v_{c} \ln r}{r^{3}}\right), & 1 /\left|v_{c}\right| \gg r \gg 1 \\
\left(\begin{array}{c}
\frac{2}{\alpha} \ln r+\text { const } \\
\mathcal{O}\left(r^{2}\right)
\end{array}\right), & r \ll 1
\end{array}\right.
$$


where the constant term is to be found from the consideration of the shortdistance physics. The solution (37) is linear in $v_{c}$ and it diverges weakly for $r \rightarrow 0$. This type of divergent solution has been considered before in Ref. [25], however the short distance cutoff of the divergence was set there to be on order of the healing length. In the next subsection we study the the fate of this divergence within the vortex core and the appearance of non-analytic behavior in $v_{c}$.

4.2.2. Solution at small distances and relation to the Laplace and the Popov equations.

Let us now construct the solution of the Gross-Pitaevskii equations at distances much smaller than the healing length. According to Eqs. (31) and (37), at $r \ll 1$ this solution can be sought for in a scaling form

$$
\Psi\left(z, z^{*}\right)=i \frac{v_{c}}{\alpha} \Upsilon\left(\frac{\alpha^{2} z}{i v_{c}} ; \frac{\alpha^{2} z^{*}}{-i v_{c}^{*}}\right) .
$$

At the distances $\left|v_{c}\right| \ll|z| \ll 1$, Eq. (38) must reproduce the solution of the Bogolyubov equation, i.e.

$$
\Upsilon\left(\xi ; \xi^{*}\right)=\xi+\frac{1}{2} \ln \xi \xi^{*} ; \quad|\xi| \gg 1 .
$$

Moreover, for distances much shorter than the healing length, $|z| \ll 1$, the wave-function can be approximated by the solution of the Laplace equation

$$
\frac{\partial^{2}}{\partial \xi \partial \xi^{*}} \Upsilon\left(\xi ; \xi^{*}\right)=0,
$$

everywhere, except for a set of measure zero. From the numerical solution we know that this set should be a cut on the complex $\xi$-plane, see Fig. 4 (a), and current conservation imposes the boundary conditions on the cut:

$$
\Upsilon\left(\xi ; \xi^{*}\right)=0 ; \quad \operatorname{Im} \xi=0 ;-2<\operatorname{Re} \xi<0 ;
$$

Equations (39) are easily solved using elliptic coordinates

$$
\xi=\cosh \zeta-1 ; \quad \xi^{*}=\cosh \zeta^{*}-1 ;
$$

Transformation (40) is the conformal mapping of the complex $\xi$-plane to the strip $\operatorname{Re} \zeta \geq 0,0 \leq \operatorname{Im} \zeta<2 \pi$, see Fig. 4. The cut corresponds to the line $\operatorname{Re} \zeta=0$.

As the transformation (40) is conformal, the Laplace equation (39b) holds its form also for the new coordinates $\zeta, \zeta^{*}$, and therefore the desired solution can be easily found:

$$
\Upsilon\left(\zeta, \zeta^{*}\right)=\frac{1}{2}\left[e^{\zeta}-e^{-\zeta^{*}}+\zeta+\zeta^{*}\right]
$$


Notice that for $|\xi| \gg 1, \zeta \simeq \ln \xi$ and the above solution reduces to (39a), while on the line of purely imaginary $\zeta$ (which maps to the cut in the $\xi$-plane) the wave function vanishes $(39 \mathrm{c})$.

Equations (41), (40), and (27), give the complete solution of Popov's equations (28) at $\sigma=1, \vec{R}_{0}=0$ everywhere except on the cut singularity. The excellent agreement with numerics is demonstrated in Fig. 1
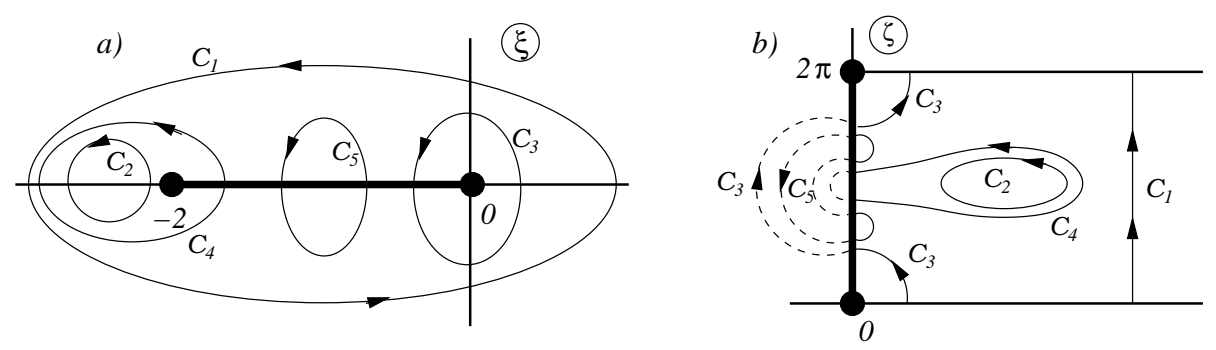

Figure 4: The conformal mapping (40) between the $\xi$-plane and a strip in the $\zeta$-plane whose lower edge should be identified with its upper edge. In the $\xi$-plane the vortex is located at the origin. Contours $\mathcal{C}_{1,3}$ encompass the vortex. The other contours do not enclose the vortex even though they may go through the cut. Dashed lines in the $\zeta$-plane corresponds to paths on the second Riemann sheet in $\xi$-plane (not shown here).

The remaining question to answer is how Eq. (41), which is fixed on the cut, reproduces the point source in the first of Eqs. (28b). To answer this question, we notice that according to (27), in all regions where $|\Upsilon|>0$

$$
-\vec{\nabla} \frac{\vec{\nabla} a_{0}}{\rho^{2}}=\vec{\nabla} \frac{\vec{E}}{\rho^{2}}=-\vec{\nabla} \hat{\varepsilon} \vec{\nabla} \theta ; \quad \theta\left(\zeta, \zeta^{*}\right)=\frac{i}{2} \ln \frac{\Upsilon^{*}\left(\zeta, \zeta^{*}\right)}{\Upsilon\left(\zeta, \zeta^{*}\right)} .
$$

This implies that $\nabla \frac{\vec{\nabla} a_{0}}{\rho^{2}}=0$ is satisfied outside the cut and only the cut region needs investigation, since on the cut $|\Upsilon|=0$, and the phase may experience an arbitrary jump. We will define this jump in the original coordinates as

$$
\begin{aligned}
& \theta(\xi+i 0)-\theta(\xi-i 0)=\theta[\zeta(\xi)]-\theta[2 \pi i-\zeta(\xi)], \\
& \operatorname{Im} \xi=0 ;-2<\operatorname{Re} \xi<0
\end{aligned}
$$

where here, in order to clarify the equation, we did not write explicitly the dependence of the phase on the conjugate variables. With the definition (43) the evolution of the phase through the cut on the $\xi$-plane, Fig. 4 a) is equivalent to the evolution along the dashed line on the $\zeta$-plane, Fig. $4 \mathrm{~b}$ ). Let us now calculate the two-dimensional integral of Eq. (42) over some region $\mathcal{A}_{i}$. We obtain

$$
\int_{\mathcal{A}_{i}} d^{2} r \vec{\nabla} \frac{\vec{E}}{\rho^{2}}=\int_{\mathcal{C}_{i}} d \vec{\ell} \vec{\nabla} \theta=\frac{1}{2} \int_{\mathcal{C}_{i}}\left[d \zeta \frac{\partial \theta}{\partial \zeta}+d \zeta^{*} \frac{\partial \theta}{\partial \zeta^{*}}\right] .
$$

Here $\mathcal{C}_{i}$ is the directed contour surrounding the area $\mathcal{A}_{i}$, and in the last equation we used the conformal transformation (40) and the definition of the phase jump 
(43). Finally, using $\theta(\zeta+2 \pi i)=\theta+2 \pi$, we obtain from inspection of Fig. 4 b), that only the contours going around point $\xi=0$ acquire the phase $2 \pi$

$$
\frac{1}{2} \int_{\mathcal{C}_{i}}\left[d \zeta \frac{\partial \theta}{\partial \zeta}+d \zeta^{*} \frac{\partial \theta}{\partial \zeta^{*}}\right]=2 \pi \int_{\mathcal{A}_{i}} d^{2} r \delta(\mathbf{r})
$$

As the area $\mathcal{A}_{i}$ is arbitrary, Eqs. (45) and (44) reproduce the point source in Eq. (28a).

\subsection{Solution at large distances and the vortex dipole moment.}

The solution at distance larger than the healing length is obtained from Eqs. (36-37) in the range $1 \ll r \ll 1 / v_{c}$, by matching Eqs. (38), (41), and (40) with the linearized asymptotics (36-37) at any $v_{c} \ll r \ll 1$. With logarithmic accuracy we find

$$
\left(\begin{array}{l}
U \\
V
\end{array}\right)=\left(\begin{array}{l}
U^{(2)} \\
V^{(2)}
\end{array}\right)+\frac{i v_{c}}{2 \alpha^{2}}\left[\ln \frac{1}{\left|v_{c}\right|}+\mathcal{O}(1)\right]\left(\begin{array}{l}
U^{(1)} \\
V^{(1)}
\end{array}\right)
$$

At $r \gg 1$, we find

$$
\left(\begin{array}{l}
U \\
V
\end{array}\right)=\frac{i v_{c}}{2}\left(\begin{array}{c}
r+\frac{\ln r}{r}+\frac{\ln \left(1 /\left|v_{c}\right|\right)}{\alpha^{2} r}+\frac{1}{2 r} \\
-r-\frac{\ln r}{r}-\frac{\ln \left(1 /\left|v_{c}\right|\right)}{\alpha^{2} r}+\frac{1}{2 r}
\end{array}\right)+\mathcal{O}\left(\frac{v_{c} \ln r}{r^{3}}\right),
$$

Substituting Eq. (46) into Eq. (31) and the result in Eq. (27), and recalling that $v=\hat{\varepsilon} E / B$, we obtain Eqs. (3-5) of the introduction. This completes the derivation of the microscopic expression of the dipole moment.

It is instructive to rewrite Eq. (3) in Galilean invariant form. Using Eqs. (24), taking into account the relation between the superfluid velocity and current at large distances $\vec{v}_{c} \simeq \hat{\varepsilon} \vec{E} / B$ and returning to the two-dimensional vector notation, we obtain for the field distribution around the vortex $\vec{R}_{i}(t)$ with vorticity $\sigma_{i}$

$$
\begin{aligned}
& \frac{\vec{E}}{B}=\frac{\vec{E}_{i}}{B_{i}}+\left[\frac{\sigma_{i} \vec{r}_{i}}{\left|\vec{r}_{i}\right|^{2}}+\frac{2\left(\vec{d}_{i} \cdot \vec{r}_{i}\right) \vec{r}_{i}-\vec{r}_{i}^{2} \vec{d}_{i}}{\left|\vec{r}_{i}\right|^{4}}+\mathcal{O}\left(\frac{\vec{v}}{r_{i}^{2}}\right)\right] ; \\
& \vec{r}_{i} \equiv \vec{r}-\vec{R}_{i}(t) ; \\
& \vec{d}_{i}(r)=\vec{f}_{i} \ln \left|\vec{r}_{i}\right|+\vec{d}(1) ; \quad \vec{d}_{i}(1)=\frac{\vec{f}_{i}}{\alpha^{2}} \ln \frac{1}{\left|\vec{f}_{i}\right|},
\end{aligned}
$$

where the force $\vec{f}_{i}$ was defined in Eq. (23), with the fields understood as assuming their asymptotic limit far from the vortex (corresponding to $E_{i}$ ). It is important to emphasize that the dipole moment as well as the force acting on vortex are Galilean invariant.

Equations (47) are the basic ingredients for our construction of an effective field theory describing physics over scales larger than the healing length. 


\section{Effective field theory at distances larger than the healing length.}

So far, we considered the solutions for a uniformly moving vortex in a stationary flow. Now we wish to describe the full dynamics of the vortices and fields in the limit of low frequency excitations. To do that we take advantage of the separation of length scales in the problem. Imagine an element of the superfluid (which may or may not include a vortex) oscillating at some frequency $\omega \ll 1$. The oscillatory nature of the motion of this element can be observed only at distances larger or of the order of $1 / \omega$. At the smaller distances the velocity field is essentially uniform and can be considered within the quasistatic approximation, for which the solution for a single vortex is known (see Sec. 3). This property allows one to write an effective theory in terms of slow fields which change over scales much larger than the healing length, provided we consider only situations where the distances between vortices are much larger than the healing length. (The interesting case of a vortex-antivortex pair at distances on order of or smaller than the healing length will be considered elsewhere [26]).

Apparently, the most straightforward way to introduce the long-wavelength effective theory is simply to drop the gradient $(\vec{\nabla} B)^{2}$ term in Eq. (19).

$$
\mathcal{S}_{\text {eff }} \stackrel{?}{=} \int d^{3} x\left[\frac{\vec{E}^{2}}{2 B}-\frac{(B-1)^{2}}{2}-2 \pi \boldsymbol{J}^{V} \cdot \boldsymbol{A}\right] .
$$

In the absence of vortex current, $\boldsymbol{J}^{V}=0$, this is a legitimate approximation. However, when $\boldsymbol{J}^{V} \neq 0$ the above action diverges due to the $\delta$-functions in the vortex density and currents (15). In other words, the local hydrodynamical description is not applicable in the vicinity of the vortex core.

In order to regularize this divergence one has to replace the point like source by a smeared source of size larger than the healing length $r_{0} \gtrsim 1$. There are many ways of smearing the $\delta$-function. We find that the most convenient one, from the technical point of view, is to replace the point-source by a ring-source of radius $r_{0}$, i.e.

$$
2 \pi \delta(\vec{r}) \rightarrow 2 \pi \delta_{r_{0}}(\vec{r}) \equiv \frac{\delta\left(|\vec{r}|-r_{0}\right)}{r_{0}} .
$$

This form ensures that the perturbation of the density due to the contribution from the vicinity of the vortex can not be larger than $1 /\left(2 r_{0}^{2}\right) \lesssim 1$.

However, the effective theory (48) with the regularization (49) still does not account for the change in the density and the action due to the contribution from the core of the $l$-th vortex ${ }^{1}$. However, this contribution is coming from short distances, and can therefore be found for each vortex separately by employing the quasistationary approximation and using the results presented in Sec. 4 which provide a full description of the vortex core.

\footnotetext{
${ }^{1}$ The above regularization implies that fields inside the ring around the $l$-th vortex are due to the external fields and the fields created by all the vortices except $l$-th one.
} 
The resulting effective action takes the form

$$
\mathcal{S}_{e f f}=\int d^{3} x\left[\frac{\vec{E}^{2}}{2 B}-\frac{(B-1)^{2}}{2}-2 \pi \tilde{\boldsymbol{J}}^{V} \cdot \boldsymbol{A}\right]+\sum_{l} \mathcal{S}^{V}\left[\vec{R}_{l}(t), \vec{f}_{l}\right],
$$

where the vortex current $\tilde{\boldsymbol{J}}^{V}$ is calculated using Eq. (15) with the regularization (49), while the last term represents the contribution from the core of each one of the vortices. This contribution is a functional of the vortex velocity, $\partial_{t} \vec{R}_{l}$, and the force, $\overrightarrow{f_{l}}$, acting on the vortex [see Eq. (23)]:

$$
\begin{aligned}
& \vec{f}_{l}(t)=\vec{E}_{l}+B_{l} \hat{\varepsilon} \partial_{t} \vec{R}_{l}(t) \\
& \left(\begin{array}{l}
\vec{E}_{l} \\
B_{l}
\end{array}\right) \equiv \int d^{2} r \delta_{r_{0}^{-}}\left[\vec{r}-\vec{R}_{l}(t)\right]\left(\begin{array}{l}
\vec{E}(\vec{r}, t) \\
B(\vec{r}, t)
\end{array}\right) .
\end{aligned}
$$

Here $\delta_{r_{0}^{-}}$is the regularized $\delta$-function defined in Eq. (49) with the limit $r_{0} \rightarrow$ $r_{0}-0^{+}$in accordance with the comment of footnote 1 .

The form of the functional $\mathcal{S}^{V}\left[\vec{R}_{l}(t), \vec{f}_{l}\right]$ is invariant under translation, gauge, and Galilean transformations. In the next section we shall show it has the form:

$$
\mathcal{S}^{V}[\vec{R}(t), \vec{f}]=\int d t\left[\frac{\pi}{\alpha^{2}} \vec{f}^{2} \ln \frac{1}{|\vec{f}|}-\Delta E(\tilde{B}(\vec{R}))\right],
$$

where the constant $\alpha=0.8204 \ldots$ is defined in Eq. (6).

The first term in the square brackets (dipole term) is the non-analytic contribution due to the force $\vec{f}$ acting on the vortex which produces the cut singularity and the dipole moment $\vec{d}$ discussed in Sec. 4. [Another way to represent the dipole term is to write $\vec{f} \cdot \vec{d}(1)$, see Eq. (47)].

The second (energy) term, $\Delta E(\tilde{B}(\vec{R}(t))$ should be understood as the contribution to the energy by the non-deformed core. One could think that it is constant and can be neglected in the action, however, this energy depends on the smooth part of the density (for example when $B \neq 1$ due to wave deformation) and will contribute in the resulting equation of motion. The "smoothened" field $\tilde{B}(\vec{R})$ can be defined as an average of the effective field $B$ inside the ring

$$
\tilde{B}(\vec{R})=\frac{1}{\pi r_{0}^{2}} \int d^{2} r \Theta\left(r_{0}-|\vec{r}-\vec{R}|\right) B(\vec{r}),
$$

where $\Theta(x)$ is the Heaviside step function.

In principle, it is possible to have an additional contribution allowed by Galilean invariance: The term $\left(\partial_{t} \vec{R}\right)^{2}$, which adds only total time derivatives to the Lagrangian under Galilean transformations. [This kinetic term can be interpreted as an additional contribution to the kinetic energy due to the fact that the true density in the vortex is lower than the one obtained by the effective theory]. However, we shall show that this term is absent within our consistent effective theory, see Secs. 5.1 and 6.1. 
To conclude the description of the effective vortex we mention the relation of the dipole term $\vec{f} \cdot \vec{d}(1)$ to the calculation of the logarithmically divergent mass of the vortex $[27,28,29,25]$. The latter approach consists of writing $\vec{d}=\vec{f} \ln \left|\vec{r}_{0}\right|$ and considering $r_{0}$ to be very large (on order of the intervortex distance) to include the whole dipole deformation of the suprefluid field in the vortex mass, thus effectively decoupling the superfluid waves and the vortex motion. Our effective theory defined on the scale $r_{0} \gtrsim 1$ includes all these contribution but treats them on the level of the deformation of the superfluid fields $\mathcal{B}, \mathcal{E}$ described by the equation of motion. However, our theory includes the previously overlooked non-analytic contribution $\vec{d}(1) \sim \vec{f} \ln (1 /|f|)$ coming from the core of the vortex. We will see later that this non-analytic contribution is decisive for the slow vortex dynamics, see Sec. 6 for more details.

\subsection{Derivation of the effective action for the vortex core.}

In this section we derive formula (52) describing the contribution to the action from the vicinity of the vortex. It is clear by construction of the effective action Eq. (50) that in order to calculate $\mathcal{S}^{V}$ it is sufficient to consider a single vortex, characterized by its coordinate $\vec{R}(t)$ and its vorticity $\sigma$.

The action $\mathcal{S}^{V}$ can be understood as the difference between the exact action, $\mathcal{S}_{\mathcal{B}, \mathcal{E}}$, and the effective action of the smooth fields, $\mathcal{S}_{B, E}$, where both contributions are evaluated within a circle of radius $r_{0}$ around the vortex position $\vec{R}$ :

$$
\mathcal{S}^{V}=\mathcal{S}_{\mathcal{B}, \mathcal{E}}-\mathcal{S}_{B, E}
$$

The exact action can be found by using the fields, $\overrightarrow{\mathcal{E}}, \overrightarrow{\mathcal{B}}$, describing the quasistationary solution of the equations of motion, while the effective action is the long-wavelength action described by the integral on the right-hand side of (50) (we reserve the $\vec{E}, B$ notation for the smooth fields introduced in this action).

The subtraction of $\mathcal{S}_{B, E}$ is designed to simply exclude the contribution of the smooth fields near the vortex core where the local description of the action is not applicable,

$$
\begin{aligned}
\mathcal{S}_{B, E} & =\int_{|\vec{r}-\vec{R}(t)|<r_{0}} d^{3} x\left[\frac{\vec{E}^{2}}{2 B}-\frac{(B-1)^{2}}{2}\right] \\
& -2 \pi \sigma \int d^{3} x \delta_{r_{0}}[\vec{r}-\vec{R}(t)]\left[a_{0}(\vec{r}, t)-\vec{a}(\vec{r}, t) \partial_{t} \vec{R}\right] .
\end{aligned}
$$

where in the last term we used explicit expressions (15) and (49) for the vortex density current $\tilde{\boldsymbol{J}}^{V}$.

To evaluate the contribution $\mathcal{S}_{\mathcal{B}, \mathcal{E}}$, it is convenient to use a coordinate system which is attached to the vortex position, $\vec{R}(t)$. Thus all the fields should be transformed to the moving (not necessarily inertial) frame according to Eq. (24) 
with $\vec{v} \rightarrow \partial_{t} \vec{R} ; \vec{v} t \rightarrow \vec{R}(t)$ :

$$
\begin{aligned}
\mathcal{S}_{\mathcal{B}, \mathcal{E}} & =\int_{|\vec{r}|<r_{0}} d^{3} x\left\{\frac{\left(\overrightarrow{\mathcal{E}}-\mathcal{B} \hat{\varepsilon} \partial_{t} \vec{R}\right)^{2}}{2 \mathcal{B}}-\left[\frac{(\mathcal{B}-1)^{2}}{2}+\frac{(\vec{\nabla} \sqrt{\mathcal{B}})^{2}}{2}\right]\right\} \\
& -2 \pi \int_{|\vec{r}|<r_{0}} d^{3} x \tilde{a}_{0} J_{0}^{V} .
\end{aligned}
$$

Galilean invariance (24) and the continuity of the fields on the ring of radius $r_{0}$ around the vortex imply that

$$
\begin{aligned}
& \left.\tilde{a}_{0}(\mathbf{r}, t)\right|_{|\mathbf{r}|=r_{0}}=\left.\left\{a_{0}[\mathbf{r}+\vec{R}(t), t]-\vec{a}[\mathbf{r}+\vec{R}(t), t] \partial_{t} \vec{R}(t)\right\}\right|_{|\mathbf{r}|=r_{0}} ; \\
& \left.\tilde{\vec{a}}(\mathbf{r}, t)\right|_{|\mathbf{r}|=r_{0}}=\left.\vec{a}[\mathbf{r}+\vec{R}(t), t]\right|_{|\mathbf{r}|=r_{0}} ; \\
& \left.\frac{\overrightarrow{\mathcal{E}}-\mathcal{B} \hat{\varepsilon} \partial_{t} \vec{R}}{\mathcal{B}}\right|_{|\mathbf{r}|=r_{0}}=\left.\frac{\vec{E}}{B}\right|_{|\mathbf{r}|=r_{0}-0^{+}}+\left.\left[\frac{\sigma \vec{r}}{|\vec{r}|^{2}}+\frac{2(\vec{d} \cdot \vec{r}) \vec{r}-\vec{r}^{2} \vec{d}}{|\vec{r}|^{4}}+\ldots\right]\right|_{|\mathbf{r}|=r_{0}},
\end{aligned}
$$

where $\tilde{a}_{0}(\mathbf{r}, t)$ and $\tilde{\vec{a}}(\mathbf{r}, t)$ denote the smooth gauge fields of the effective action, and we use the definition $|\mathbf{r}|=r_{0}-0^{+}$for the smooth fields (see footnote 1 ). The last equation is obtained using the dipole expansion for the field outside the vortex (47).

We now turn to calculate of the contribution $\mathcal{S}_{\mathcal{B}, \mathcal{E}}$, which is a functional of the quasi-static fields. Consider, first, the source term in (the second line of) (54c). Using the equation of motion (22b), and a Galilean transformation to the coordinate system comoving with the vortex (24), we can rewrite it as

$$
-2 \pi \int_{|\vec{r}|<r_{0}} d^{3} x \tilde{a}_{0} J_{0}^{V}=-\int_{|\vec{r}|<r_{0}} d^{3} x \tilde{a}_{0} \vec{\nabla} \cdot\left(\frac{\overrightarrow{\mathcal{E}}-\mathcal{B} \hat{\varepsilon} \partial_{t} \vec{R}}{\mathcal{B}}\right) .
$$

Integrating by parts and utilizing the connection between the fields and vector potential (20), we obtain

$$
\begin{aligned}
-2 \pi \int_{|\vec{r}|<r_{0}} d^{3} x \tilde{a}_{0} J_{0}^{V} & =-\int d t \oint_{|\vec{r}|=r_{0}} \tilde{a}_{0}\left[\left(\frac{\overrightarrow{\mathcal{E}}-\mathcal{B} \hat{\varepsilon} \partial_{t} \vec{R}}{\mathcal{B}}\right) \hat{\varepsilon} d \vec{r}\right] \\
& -\int_{|\vec{r}|<r_{0}} d^{3} x\left(\frac{\overrightarrow{\mathcal{E}}-\mathcal{B} \hat{\varepsilon} \partial_{t} \vec{R}}{\mathcal{B}}\right)\left(\overrightarrow{\mathcal{E}}+\partial_{t} \overrightarrow{\tilde{a}}\right) .
\end{aligned}
$$

Here, and henceforth, the vector $d \vec{r}$ appearing in the surface terms, is directed along the contour. We now eliminate the time derivative of the vector potential via integration by parts and the equation of motion $(22 \mathrm{c})$ :

$$
\partial_{t}\left(\frac{\overrightarrow{\mathcal{E}}-\mathcal{B} \hat{\varepsilon} \partial_{t} \vec{R}}{\mathcal{B}}\right)=\hat{\varepsilon} \vec{\nabla} \mathcal{H} ; \quad \mathcal{H} \equiv\left(\frac{\overrightarrow{\mathcal{E}}^{2}}{2 \mathcal{B}^{2}}+B-1-\frac{1}{2 \sqrt{\mathcal{B}}} \vec{\nabla}^{2} \sqrt{\mathcal{B}}\right) .
$$


This reduces Eq. (55) to

$$
\begin{aligned}
& -2 \pi \int_{|\vec{r}|<r_{0}} d^{3} x \tilde{a}_{0} J_{0}^{V}=-\int d t \oint_{|\vec{r}|=r_{0}} \tilde{a}_{0}\left[\left(\frac{\overrightarrow{\mathcal{E}}-\mathcal{B} \hat{\varepsilon} \partial_{t} \vec{R}}{\mathcal{B}}\right) \hat{\varepsilon} d \vec{r}\right] \\
& -\int_{|\vec{r}|<r_{0}} d^{3} x\left[\left(\frac{\overrightarrow{\mathcal{E}}-\mathcal{B} \hat{\varepsilon} \partial_{t} \vec{R}}{\mathcal{B}}\right) \overrightarrow{\mathcal{E}}-\overrightarrow{\tilde{a}} \hat{\varepsilon} \vec{\nabla} \mathcal{H}\right]
\end{aligned}
$$

Integrating the last term by parts and using $\vec{\nabla} \hat{\varepsilon} \overrightarrow{\tilde{a}}=\mathcal{B}$, we arrive at an expression which separates the contribution from the interior of the vortex core [denoted below by $(b)]$, from the surface terms coming from the interface between the core the exterior of the vortex [denoted by $(s)]$ :

$$
\begin{aligned}
& -2 \pi \int_{|\vec{r}|<r_{0}} d^{3} x \tilde{a}_{0} J_{0}^{V}=\Sigma_{b}+\Sigma_{s} ; \\
& \Sigma_{b}=\int_{|\vec{r}|<r_{0}} d^{3} x\left[-\left(\frac{\overrightarrow{\mathcal{E}}-\mathcal{B} \hat{\varepsilon} \partial_{t} \vec{R}}{\mathcal{B}}\right) \overrightarrow{\mathcal{E}}+\mathcal{B H}\right] \\
& \Sigma_{s}=-\int d t \oint_{|\vec{r}|=r_{0}}\left[\frac{\tilde{a}_{0}\left(\overrightarrow{\mathcal{E}}-\mathcal{B} \hat{\varepsilon} \partial_{t} \vec{R}\right) \hat{\varepsilon}}{\mathcal{B}}+\mathcal{H} \tilde{\vec{a}}\right] d \vec{r} .
\end{aligned}
$$

The surface contribution $\Sigma_{s}$ can be now evaluated using the asymptotic expression of the fields at $r=r_{0}$, see Eq. (54d). With the same accuracy of this asymptotic expansion we find from Eq. (56)

$$
\left.\mathcal{H}\right|_{|\vec{r}|=r_{0}}=-\left.\partial_{t}\left(\frac{\vec{r} \hat{\varepsilon} \vec{E}}{B}\right)\right|_{|\vec{r}|=r_{0}-0^{+}}-\left.\partial_{t}\left[\frac{\vec{r} \hat{\varepsilon} \vec{d}\left(t, r_{0}\right)}{r^{2}}\right]\right|_{|\vec{r}|=r_{0}} .
$$

We substitute Eqs. (54d) and (59) into Eq. (58), and use the following relations satisfied by an arbitrary smooth function $b(\vec{r})$

$$
\begin{aligned}
& \oint_{\left|r_{1}\right|=r_{0}} \frac{\overrightarrow{r_{1}} \hat{\varepsilon} d \vec{r}_{1}}{\left|\overrightarrow{r_{1}}\right|^{2}} b\left(\vec{r}+\vec{r}_{1}\right)=\oint_{|r|_{1}=r_{0}} \frac{d r_{1}}{r_{1}} b\left(\vec{r}+\vec{r}_{1}\right)=2 \pi \int d^{2} r_{1} \delta_{r_{0}}\left(\vec{r}-\vec{r}_{1}\right) b\left(\vec{r}_{1}\right) ; \\
& \oint_{\left|r_{1}\right|=r_{0}} \frac{\left[2 \vec{r}_{1}\left(\vec{d} \cdot \vec{r}_{1}\right)-\vec{d} \vec{r}_{1}^{2}\right] \hat{\varepsilon} d \vec{r}_{1}}{\left|\vec{r}_{1}\right|^{4}} b\left(\vec{r}+\vec{r}_{1}\right) \simeq \pi \int d^{2} r_{1} \delta_{r_{0}}\left(\vec{r}-\vec{r}_{1}\right) \vec{d} \cdot \vec{\nabla} b\left(\vec{r}_{1}\right) ;
\end{aligned}
$$

where the smeared $\delta$-function is defined in Eq. (49). We obtain

$$
\begin{aligned}
\Sigma_{s} & =\int d^{3} x \delta_{r_{0}^{-}}[\vec{r}-R(t)] \\
& \times\left\{\pi r_{0}^{2}\left[\frac{\vec{E}^{2}}{2 B}\right]_{t, \vec{r}}-2 \pi \sigma\left[a_{0}-\vec{a} \partial_{t} \vec{R}\right]_{t, \vec{r}}+\pi \vec{d} \cdot\left[\vec{E}+B \hat{\varepsilon} \partial_{t} \vec{R}\right]_{t, \vec{r}}\right\},
\end{aligned}
$$


and the smeared $\delta$-function, $\delta_{r_{0}^{-}}(\vec{r})$ is defined by Eq. (49) with $r_{0} \rightarrow r_{0}-0^{+}$.

We complete the derivation by substituting Eq. (61) into Eq. (58) and plugging the result into Eqs. (54). Performing analogous transformations, neglecting the boundary term $\vec{r} \vec{\nabla} B$ at $|\vec{r}|=r_{0}$, and using the expression (4) for the dipole moment, we obtain ${ }^{2}$

$$
\mathcal{S}^{V}=\int d t\left[\mathcal{L}_{d}+\mathcal{L}_{K}-\Delta E\right]
$$

The dipole Lagrangian is the main result of this section as it describes a contribution which is non-analytic in the applied force,

$$
\mathcal{L}_{d}=\frac{\pi \vec{f}^{2} \ln \frac{1}{|\vec{f}|}}{\alpha^{2}}
$$

where the force acting on vortex was defined in Eq. (51). Needless to say the dipole Lagrangian is Galilean invariant as the force acting on vortex $2 \pi \sigma \vec{f}$ is Galilean invariant.

The term $\Delta E$ is simply the contribution of the non-deformed vortex core to the energy:

$$
\Delta E=\int_{|\vec{r}-\vec{R}(t)|<r_{0}} d^{2} r\left[\frac{1-\mathcal{B}^{2}}{2}-\frac{(B-1)^{2}}{2}\right],
$$

Finally, the term $\mathcal{L}_{K}$ describes the analytic velocity dependence of the energy of the vortex. Its formal expression is

$$
\mathcal{L}_{K}=\int_{|\vec{r}-\vec{R}(t)|<r_{0}}\left[\frac{\mathcal{B}\left(\partial_{t} \vec{R}\right)^{2}}{2}-\frac{\vec{E}^{2}}{2 B}+\int d^{2} r_{1} \delta_{r_{0}^{-}}\left(\vec{r}_{1}-\vec{R}\right)\left(\frac{\vec{E}^{2}}{2 B}\right)_{\vec{r}_{1}}\right] ;
$$

For homogeneous fields the last two terms cancel each other, so to explicitly calculate this term would require us to investigate the internal structure of the moving vortex within the effective theory. Such study is, however, not necessary as the form of $\mathcal{L}_{K}$ can be deduced from the Galilean invariance. The latter dictates that $\mathcal{L}_{K}$ can include only combinations of $\left(\partial_{t} R\right)^{2}$ (as it adds only total a time derivative to the Lagrangian under Galilean transformations) and any analytic function of $\vec{f}^{2}$. However, an analytic function of $\vec{f}^{2}$ is negligible in comparison with non-analytic dipole Lagrangian (62b), and therefore

$$
\mathcal{L}_{K}=\beta\left(\partial_{t} \vec{R}\right)^{2},
$$

where $\beta$ is a coefficient of order one which can be found using Galilean invariance once again. Indeed, starting with a system of vortices at rest and switching to a coordinate frame moving with the velocity $\vec{v}$, dipole moments in this case do

\footnotetext{
${ }^{2}$ With the desired accuracy $\vec{d}\left(r_{0}\right)=\vec{d}(1)$.
} 
not arise and the terms $(62 \mathrm{~b})$ and $(62 \mathrm{c})$ do not change the action. On the other hand, the original action transforms according to Eq. (25), i.e. the coefficient in Eq. (62d) is just the difference between the number of particles in the effective stationary vortex core and true stationary vortex core:

$$
\beta=\int_{|\vec{r}-\vec{R}|<r_{0}} d^{2} r[\mathcal{B}-B] .
$$

We will show in Sec. 6.1, that the density profile in effective theory is arranged to exactly reproduce the same number of particles in the vortex core as the microscopic solution, so that $\beta=0$. The action (52) follows from Eqs. (62).

\subsection{The equations of motion.}

The derivation of the equations of motion resulting from Eqs. (50-53) is analogous to the derivation of Eqs. (22). Because Eq. (50) is a gauge theory, the first Maxwell equation is still valid,

$$
\partial_{t} B=-\vec{\nabla} \times \vec{E}
$$

Instead of Eq. (22b) we obtain

$$
\vec{\nabla} \cdot\left(\frac{\vec{E}}{B}\right)=2 \pi \tilde{J}_{0}^{V}-\vec{\nabla} \cdot \overrightarrow{\mathcal{P}}_{V}
$$

where the smoothened vortex density-current is calculated using Eq. (15) with the regularization (49), corresponding to a simple smoothening of the original action. The last term, new in comparison with Eq. (22b), is the change in vortex density due to polarization of the vortex shape by an external force:

$$
\overrightarrow{\mathcal{P}}_{V}=\sum_{l} \delta_{r_{0}^{-}}\left[\vec{r}-\vec{R}_{l}(t)\right] \vec{d}_{l} ; \quad \vec{d}_{l}=\frac{\partial}{\partial f_{l}}\left(\frac{\pi}{\alpha^{2}} \vec{f}_{l}^{2} \ln \frac{1}{\left|\vec{f}_{l}\right|}\right)
$$

see Eqs. (51), (49) for definitions of the force $\overrightarrow{f_{l}}$ and the smoothen function $\delta_{r_{0}^{-}}$. Equation $(63 \mathrm{~b})$ is analogous to the generalization of electrodynamics for polarizable media.

Equation (22c) is replaced by

$$
\hat{\varepsilon} \vec{\nabla}\left[\frac{1}{2}\left(\frac{\vec{E}}{B}\right)^{2}+B\right]=\hat{\varepsilon} \vec{\nabla} \mathcal{M}+\vec{j}_{v}+\partial_{t}\left(\frac{\vec{E}}{B}+\overrightarrow{\mathcal{P}}_{v}\right)
$$

i.e. polarization and magnetization currents are now added to the vortex current.

The magnetic moment density is the derivative of the effective action (52) with respect to local magnetic field. We present it as the sum of two parts

$$
\mathcal{M}=\sum_{l}\left\{\delta_{r_{0}^{-}}\left[\vec{r}-\vec{R}_{l}(t)\right] \mathfrak{m}_{l}^{(d)}+\frac{\Theta\left(r_{0}-\left|\vec{r}-\vec{R}_{l}\right|\right)}{\pi r_{0}^{2}} \mathfrak{m}_{l}^{(c)}\right\} .
$$


The first term is the magnetic moment arising from the motion of the cut singularity:

$$
\mathfrak{m}_{l}^{(d)}=-\frac{\partial}{\partial B_{l}} \frac{\pi}{\alpha^{2}} \vec{f}_{l}^{2} \ln \frac{1}{\left|\overrightarrow{f_{l}}\right|},
$$

the second term is the magnetization due to the circular currents in the nonperturbed core:

$$
\mathfrak{m}_{l}^{(c)}=-\frac{\partial \Delta E\left(B_{l}\right)}{\partial B_{l}} .
$$

Finally, we derive an equation of motion for the vortex. Varying Eqs. (5052 ) with respect to the vortex coordinate $\vec{R}_{l}(t)$ we obtain instead of Eq. (23)

$$
\frac{d}{d t} \frac{\partial}{\partial\left(\partial_{t} \vec{R}_{l}\right)}\left(\frac{\pi}{\alpha^{2}} \vec{f}_{l}^{2} \ln \frac{1}{\left|\vec{f}_{l}\right|}\right)=2 \pi \sigma_{l} \vec{f}_{l}+\frac{\partial}{\partial \vec{R}_{l}}\left(\frac{\pi}{\alpha^{2}} \vec{f}_{l}^{2} \ln \frac{1}{\left|\vec{f}_{l}\right|}\right)+\mathfrak{m}_{l}^{(c)} \frac{\partial B_{l}}{\partial \vec{R}_{l}} .
$$

As opposed to Eq. (23), equation (64) describes the complete vortex dynamics with second time derivatives generated by the interaction with the phonon field.

It is worth emphasizing that Eqs. (63) and (64) are Galilean invariant by construction. In the next section we will solve the classical equations of motion for the most important physical situations.

\section{Solutions of the classical equations of motion.}

6.1. The effective theory of a single vortex and calculation of constants in the effective action.

Let us begin with the consideration of a free vortex located at the origin, $\vec{R}=0$, in the framework of the effective equations of motion (63). The purpose of this study is to compare the resulting vortex shape with the exact one and find the expressions for the core energy $\Delta E$ and for the coefficient $\beta$ of Eq. (62e). As the problem is axially symmetric, $\vec{\nabla} \hat{\varepsilon} \vec{E}=0$ automatically, $\vec{f}=0$, and Eq. (63b) takes the form

$$
\vec{\nabla} \cdot\left(\frac{\vec{E}}{B}\right)=\frac{\sigma}{r_{0}} \delta\left(|\vec{r}|-r_{0}\right),
$$

with the obvious solution

$$
\frac{\vec{E}}{B}=\frac{\sigma \vec{r}}{|\vec{r}|^{2}} \Theta\left(|\vec{r}|-r_{0}\right)
$$

where $\Theta(x)$ is the step function. Integrating Eq. (63d) to obtain the Bernoulli form, and substituting Eq. (65) into the result, we find the magnetic field, $B(r)$, of the effective theory

$$
B(r)=B_{\infty}-\frac{\Theta\left(r-r_{0}\right)}{2 r^{2}}-\frac{\Theta\left(r_{0}-r\right)}{\pi r_{0}^{2}} \frac{\partial \Delta E}{\partial B_{\infty}} .
$$


Thus effective theory reproduces correctly the leading asymptotic behavior in the exterior domain $r>r_{0}$ (up to integrable terms). In the interior domain, $r<$ $r_{0}$, the effective theory may either overestimate or underestimate the density, see Fig. 5. This difference will manifest itself in the value of the coefficient $\beta$, as follows from Eq. (62e). Using $B(r)$ from Eq. (66) and utilizing the axial symmetry we find

$$
\beta=2 \pi \int_{0}^{r_{0}} d r r\left[\mathcal{B}-B_{\infty}\right]+\frac{\partial \Delta E}{\partial B_{\infty}}
$$

The energy of the core $\Delta E$ with the condition $B(r \gg 1) \rightarrow B_{\infty}$, and the field inside the vortex $\mathcal{B}$ can be found from the minimum of the energy functional

$$
\begin{aligned}
& \frac{\delta \Delta E\left[B_{\infty}, \mathcal{B}(r)\right]}{\delta \mathcal{B}(r)}=0 \\
& \Delta E\left[B_{\infty}, \mathcal{B}(r)\right]=2 \pi \int_{0}^{r_{0}} d r r\left[\frac{\mathcal{B}}{2 r^{2}}+\frac{1}{2}\left(\frac{\sqrt{\mathcal{B}}}{d r}\right)^{2}+\frac{\left(\mathcal{B}-B_{\infty}\right)^{2}}{2}\right] .
\end{aligned}
$$

From here it follows that

$$
\frac{\partial \Delta E}{\partial B_{\infty}}=2 \pi \int_{0}^{r_{0}} d r r\left(B_{\infty}-\mathcal{B}\right)
$$

and substituting in Eq. (67), we obtain

$$
\beta=0
$$

This result implies that our effective theory not only correctly describes the density $B$ outside the vortex core but also conserves the number of particle inside the core of the vortex. Therefore, the Galilean invariance is preserved automatically and there is no need for the extra term $\left(\partial_{t} \vec{R}\right)^{2}$ in order to preserve Galilean invariance (25).

\subsection{Classical oscillatory motion of the vortex in the frozen background approx- imation}

The frozen background approximation corresponds to fixing $B=B(t)$, and $\vec{E}=\vec{E}(t)$ to be explicitly independent of the vortex position, $\vec{R}(t)$. In this case Eq. (64) reduces to

$$
\frac{d}{d t} \frac{\partial}{\partial \vec{f}}\left(\frac{\vec{f}^{2}}{2 \alpha^{2}} \ln \frac{1}{|\vec{f}|}\right)+\hat{\varepsilon} \vec{f}=0 ; \quad \vec{f}=\left[\vec{E}(t)+B(t) \hat{\varepsilon} \partial_{t} \vec{R}(t)\right]
$$

where without loss of generality we also choose $\sigma=1$.

Equation (70) describes the motion of the vortex decoupled from the rest of the superfluid in a very particular way: If some flow $\vec{E}(t)$, constant in space, is applied to the system, the vortex is displaced to a new equilibrium position 


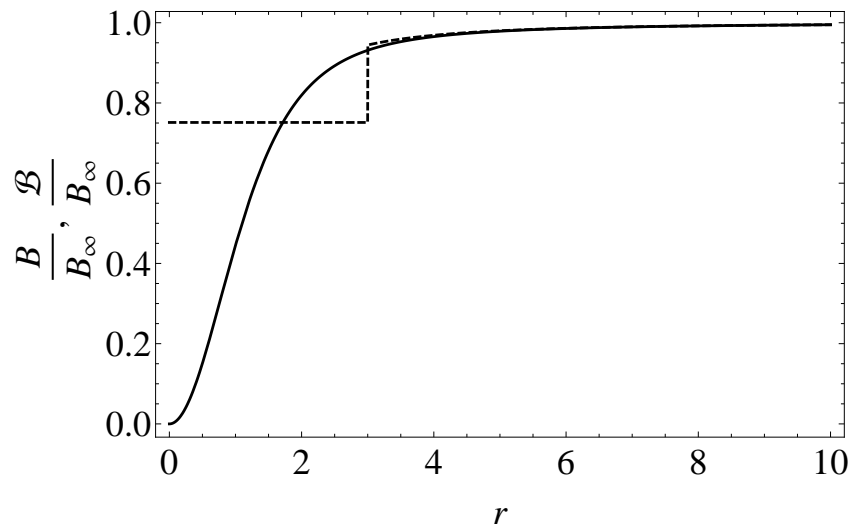

Figure 5: Comparing of the particle density $B$ in the effective theory (dashed line) and the exact form (solid line)

such that the force $2 \pi \vec{f}$ acting on the vortex remains intact. In this sense the dynamics of the force is decoupled from the rest of the superfluid, (for the origin of the residual coupling and its physical consequences see Secs. 6.3.2 and 6.3.3).

Equation (70) has an obvious solution

$$
f_{+}=f_{x}+i f_{y}=F e^{i \Omega_{c} t} ; \quad f_{-} \equiv f_{x}-i f_{y}=F^{*} e^{-i \Omega_{c} t},
$$

where the effective "cyclotron frequency" is given by

$$
\Omega_{c}(|F|)=\frac{\alpha^{2}}{\ln \frac{1}{|F|}-\frac{1}{2}} \ll 1 .
$$

Equation (72) is the main result of this section. Indeed, previous studies, see e.g. Refs. [27, 28, 29, 25], indicated that allowing for a vortex mass in the equation of motion (thereby allowing for a non-vanishing force acting on the vortex) may produce only oscillations with frequency of the order of the healing frequency $\hbar /\left(m \xi^{2}\right)$ (one in our units) which is beyond applicability of the local hydrodynamics. However, from Eq. (72) it follows that the non-analytic reconstruction of the vortex core produces an oscillation frequency which is much smaller than one, and therefore relevant also for the phonon dynamics. Moreover, the oscillation frequency $\Omega_{c}(|F|)$ depends in a non-linear manner on the amplitude of the oscillation $F=\Omega_{c} R$ and vanishes when $R \rightarrow 0$. Thus the lower limit on oscillation frequency is governed only by quantum fluctuations, See Sec. 7.

\subsection{Coupling the vortex motion to phonons}

In the remainder of this section we will study the residual coupling of a moving vortex to sound waves (phonons) in the superfluid. The main effects 
are: (i) emission of sound waves by the oscillating vortex, see Eq. (71), leading to cyclotron radiation of phonons and to a radiation reaction force on the vortex; and (ii) elastic scattering of phonons by the oscillating vortex.

Both effects are most conveniently studied in the basis of the eigenfunctions of the Popov equations (63) linearized with respect to the solution of the motionless vortex (65) - (66). We discuss partial waves solutions of this linearized equation and the corresponding scattering phases are discussed in subsection 6.3.1. Then, in the following subsections, we employ the partial wave expansion to study the radiation and elastic scattering problems.

\subsubsection{Partial wave expansion outside and inside the effective vortex core}

We linearize Eqs. $(65-66)$ with respect to the motionless vortex solution, fixing it at $\vec{R}=0$. For $|\vec{r}| \neq r_{0}$ any solution of Eq. (63b) can be parameterized as

$$
\frac{\vec{E}}{B}=\frac{\sigma \vec{r}}{|\vec{r}|^{2}} \Theta\left(|\vec{r}|-r_{0}\right)-\hat{\varepsilon} \vec{\nabla} \theta(\vec{r}, t),
$$

where the potential $\theta(\vec{r}, t)$ is to be found from the other Popov equations. Substituting Eq. (73) into Eq. (63d) and linearizing with respect to $\vec{\nabla} \theta$ we find for the exterior domain of the effective vortex core, $|\vec{r}|>r_{0}$,

$$
B(\vec{r}, t)=1-\frac{1}{2 r^{2}}+\frac{\sigma \vec{r}}{|\vec{r}|^{2}} \hat{\varepsilon} \vec{\nabla} \theta-\partial_{t} \theta
$$

This equation replaces the Bernoulli equation for the non-stationary case. In the interior domain, $|\vec{r}|<r_{0}$, the same procedure yields

$$
B(\vec{r}, t)=1-\left.\frac{1}{\pi r_{0}^{2}} \frac{\partial \Delta E}{\partial B_{\infty}}\right|_{B_{\infty}=1}-\partial_{t} \theta
$$

instead of Eq. (66).

Equations (73) and (74) enable us to find the electric field of the vortex in the framework of the effective theory. Keeping only terms linear in $\vec{\nabla} \theta$ and $\partial_{t} \theta$, we obtain

$$
\vec{E}=\frac{\sigma \vec{r}}{r^{2}}\left[1-\frac{1}{2 r^{2}}\right]-\frac{\sigma \vec{r}}{r^{2}} \partial_{t} \theta-\hat{\varepsilon} \vec{\nabla} \theta+\frac{\vec{r}^{2}+2 \vec{r} \otimes \vec{r}}{2|\vec{r}|^{4}} \hat{\varepsilon} \vec{\nabla} \theta .
$$

for $|\vec{r}|>r_{0}$. The last term in this equation will be of no importance for future manipulations and we bring it here for completeness only. Similarly, in the region $|\vec{r}|<r_{0}$ we find

$$
\vec{E}=-\left[1-\left.\frac{1}{\pi r_{0}^{2}} \frac{\partial \Delta E}{\partial B_{\infty}}\right|_{B_{\infty}=1}\right] \hat{\varepsilon} \vec{\nabla} \theta
$$

The equation for the potential, $\theta$, is now obtained by substituting Eqs. (74) and (75) into the first Maxwell equation (63a). In the exterior domain, $|\vec{r}|>r_{0}$, 
it yields

$$
\left[\vec{\nabla}^{2}-\partial_{t}^{2}+\frac{2 \sigma \vec{r}}{|\vec{r}|^{2}} \hat{\varepsilon} \vec{\nabla} \partial_{t}\right] \theta=-\hat{\varepsilon} \vec{\nabla} \frac{\vec{r}^{2}+2 \vec{r} \otimes \vec{r}}{2|\vec{r}|^{4}} \hat{\varepsilon} \vec{\nabla} \theta .
$$

One can see, by power counting, that for $|\vec{r}| \gg 1$ the right hand side of Eq. (76a) is not important and can be neglected.

Within the interior domain, $|\vec{r}|<r_{0}$, we make use of our working assumption that all relevant frequencies are much smaller than one. This allows us to write the the first Maxwell equation in the quasistatic approximation, i.e

$$
\vec{\nabla}^{2} \theta=0 .
$$

Equations (76) are axially symmetric and can be decomposed into partial waves

$$
\theta(\vec{r}, t)=\sum_{\nu, \omega} e^{-i \omega t}\left(\frac{x+i \sigma y}{r}\right)^{\nu} \theta_{\omega, \nu}(r) ; \quad \theta_{\omega, \nu}=\theta_{-\omega,-\nu}^{*} .
$$

Then, Eqs. (76) acquire the form of the Bessel equation

$$
\left[-\left(\frac{d^{2}}{d r^{2}}+\frac{d}{r d r}\right)+\frac{\nu^{2}-2 \nu \omega}{r^{2}}+\omega^{2}\right] \theta_{\omega, \nu}=0 \quad \text { for } \quad|\vec{r}|>r_{0},
$$

while

$$
\theta_{\omega, \nu} \propto r^{|\nu|} \quad \text { for } \quad|\vec{r}|<r_{0} .
$$

The choice of the particular form of the solution of Eq. (78a) depends on the problem at hand (here we assume that $\omega>0$ ). If one is interested in the problem of the cyclotron radiation by the rotating vortex, the relevant choice should have outgoing wave asymptotics i.e.

$$
\theta_{\omega, \nu}(r) \propto H_{|\nu|-\omega \operatorname{sgn} \nu}^{(1)}(\omega r),
$$

where $H_{n}^{(1)}$, is the Hankel function of $n$-th order of the first kind. (We use $\omega \ll 1$ for the calculation of the order of the Hankel function). On the other hand, if one seeks to describe the elastic scattering of phonons from a vortex, the relevant solution has the form

$$
\theta_{\omega, \nu}(r) \propto\left[J_{|\nu|-\omega \operatorname{sgn} \nu}(\omega r) \cos \tilde{\gamma}_{\nu}-Y_{|\nu|-\omega \operatorname{sgn} \nu}(\omega r) \sin \tilde{\gamma}_{\nu}\right],
$$

where $J_{n}$, and $Y_{n}$ are the Bessel and Neumann function of $n$-th order, respectively, and the phases $\tilde{\gamma}_{\nu}(\omega)$ are to be determined from the boundary condition on the circle $|\vec{r}|=r_{0}$.

The asymptotic behaviour of the solution (79) at $r \omega \gg|\nu|+1$ is

$$
\theta_{\omega, \nu}(r) \propto \sqrt{\frac{2}{\pi r \omega}} \cos \left[r \omega+\gamma_{\nu}(\omega)-\frac{\pi|\nu|}{2}-\frac{\pi}{4}\right],
$$

where the scattering phase shift is given by

$$
\gamma_{\nu}(\omega)=\frac{\pi \omega \operatorname{sgn} \nu}{2}+\tilde{\gamma}_{\nu}(\omega)
$$


The first term is a phase shift due to the Aharonov-Bohm flux induced by the vortex current (see. e.g. Ref. [30]) which does not depend on the internal state of the vortex. The second term describes the effect of the vortex core on the phonon scatterings. We will show in Sec. 6.3.3 that this phase includes a resonance associated with periodic motion of the vortex (71-72).

The expressions for the relevant cross-sections in terms of the phase shifts are well known and we do not reproduce them here.

To consider the scattering and the radiation problems we must use the equations of motion for the fields, with sources that are formally singular on the circle $|\vec{r}|=r_{0}$. To avoid this complication, it is convenient to rewrite the first Maxwell equation (63a) at $|\vec{r}|=r_{0}$ as a matching condition on the solutions inside, $|\vec{r}|=r_{0}-0^{+}$, and outside, $|\vec{r}|=r_{0}+0^{+}$, of the effective vortex core.

The first requirement is that the physical current $\hat{\varepsilon} \vec{E}$ (we can choose the current component normal to the contour $|\vec{r}|=r_{0}$ ), does not diverge. This condition can be written in integral form:

$$
\int_{\vec{r}_{-}}^{\vec{r}_{+}} d \vec{r} \hat{\varepsilon} \vec{E}(\vec{r}, t)=0 ; \quad \vec{r}_{ \pm}=\left(r_{0} \pm 0^{+}\right) \hat{r}
$$

where $\hat{r}$ is a unit vector in the direction of $\vec{r}$. Here, and in what follows, the line integration is assumed to be along the straight line connecting the endpoints $\vec{r}_{ \pm}$. To obtain the second boundary condition we substitute

$$
\vec{\nabla} \hat{\varepsilon} \vec{E}=\frac{\vec{r}}{|\vec{r}|^{2}}[\vec{\nabla}(\vec{r} \hat{\varepsilon} \vec{E})-\hat{\varepsilon} \vec{\nabla}(\vec{r} \cdot \vec{E})]
$$

into the Maxwell equation (63a) and integrate the result between the points $\vec{r}_{ \pm}$ defined in Eq. (82a), yielding:

$$
\left.(\vec{r} \hat{\varepsilon} \vec{E})\right|_{\vec{r}_{+}}-\left.(\vec{r} \hat{\varepsilon} \vec{E})\right|_{\vec{r}_{-}}=\int_{\vec{r}_{-}}^{\vec{r}_{+}} d \vec{r}\left[\hat{\varepsilon} \vec{\nabla}(\vec{r} \cdot \vec{E})-\vec{r} \partial_{t} B\right] .
$$

Next we apply the partial wave expansion to study the effects of the coupling of the vortex motion to the environment.

\subsubsection{Emission of phonons by an oscillating vortex, and the resulting decay rate.}

Let us consider a vortex with $\sigma=1$ experiencing weak oscillations around the origin $|\vec{R}(t)| \ll 1$. Linearizing the right-hand-side of Eq. (63b) with respect to small $\vec{R}(t)$ yields:

$$
\vec{\nabla} \cdot\left(\frac{\vec{E}}{B}\right)=(2 \pi-\overrightarrow{\mathbb{D}}(t) \cdot \vec{\nabla}) \delta_{r_{0}}(\vec{r}),
$$

where $\delta_{r_{0}}$ is the smeared $\delta$-function defined in Eq. (49), and the total dipole moment [compare with Eq. (63c)],

$$
\overrightarrow{\mathbb{D}}(t) \equiv 2 \pi \vec{R}(t)+\frac{\partial}{\partial \vec{f}}\left(\frac{\pi \vec{f}^{2}}{\alpha^{2}} \ln \frac{1}{|\vec{f}|}\right)
$$


perturbs the surrounding field due to the displacement of the vortex itself (the first term) to which is added the non-analytic deformation of the vortex core (the second term). Clearly, these effects are additive within the framework of a linear theory.

In analogy with our introductory remarks, (see Eq. (73)), we look for a solution in the form

$$
\frac{\vec{E}}{B}=\frac{\vec{r}}{|\vec{r}|^{2}} \Theta\left(|\vec{r}|-r_{0}\right)-\overrightarrow{\mathbb{D}}(t) \delta_{r_{0}^{-}}(\vec{r})-\hat{\varepsilon} \vec{\nabla} \theta(\vec{r}, t) .
$$

To find the source term for the density $B$ we use Eq. (63d). We notice that the magnetic moment contribution due to the vortex motion (63f) vanishes to linear order in $\vec{R}(t)$, so that

$$
\mathcal{M}(\vec{r}, t)=\left(\frac{\Theta\left(r_{0}-|\vec{r}|\right)}{\pi r_{0}^{2}}+\frac{2 \vec{R}(t) \cdot \vec{r}}{r_{0}^{2}} \delta_{r_{0}}(\vec{r})\right) \mathfrak{m}^{(c)},
$$

where the core contribution to the magnetic moment, $\mathfrak{m}^{(c)}$, is given by Eq. (63g). Substituting Eqs. (85) and (86) into Eq. (63d) yields

$$
\begin{aligned}
B(\vec{r}, t)= & -\frac{\Theta\left(|\vec{r}|-r_{0}\right)}{2 r^{2}}-\left.\frac{\Theta\left(r_{0}-|\vec{r}|\right)}{\pi r_{0}^{2}} \mu\left(B_{\infty}\right)\right|_{B_{\infty}=1} \\
& -\partial_{t} \theta+\frac{\Theta\left(|\vec{r}|-r_{0}\right) \vec{r}}{|\vec{r}|^{2}} \hat{\varepsilon} \vec{\nabla} \theta \\
& -\delta_{r_{0}^{-}}(\vec{r}) \frac{2 \vec{R}(t) \cdot \vec{r}}{r_{0}^{2}} \mathfrak{m}^{(c)} .
\end{aligned}
$$

This equation replaces Eqs. (74). The third line in Eq. (87) represents the singular source due to the motion of the vortex. From what follows it will become clear that this magnetic moment term is responsible for the coupling of the vortex motion to the environment.

Using Eqs. (85) and (87) we can identify the source term in the equation for the electric field. Neglecting terms smaller by $1 / r_{0}^{2}$ than unity [such as the last term in Eq. (75a)], we get

$$
\begin{aligned}
\vec{E}= & \frac{\Theta\left(|\vec{r}|-r_{0}\right) \vec{r}}{r^{2}}\left[1-\frac{1}{2 r^{2}}\right] \\
& -\frac{\Theta\left(|\vec{r}|-r_{0}\right) \vec{r}}{r^{2}} \partial_{t} \theta-\hat{\varepsilon} \vec{\nabla} \theta \\
& -\overrightarrow{\mathbb{D}}(t) \delta_{r_{0}^{-}}(\vec{r}) .
\end{aligned}
$$

Now, we are ready to obtain the modification of boundary conditions (82) for the field $\theta$ on the circle $|\vec{r}|=r_{0}$. Substituting Eq. (88) into Eq. (82a) yields

$$
\theta\left(\vec{r}_{+}\right)-\theta\left(\vec{r}_{-}\right)=\frac{\vec{r}_{+} \hat{\varepsilon} \overrightarrow{\mathbb{D}}(t)}{\left|\vec{r}_{+}\right|^{2}} .
$$


Similarly, substituting Eqs. (88) and (87) in Eq. (82b) yields

$$
\left.(\vec{r} \vec{\nabla} \theta)\right|_{\vec{r}_{+}}-\left.(\vec{r} \vec{\nabla} \theta)\right|_{\vec{r}_{-}}=-\frac{\vec{r}_{+} \hat{\varepsilon} \overrightarrow{\mathbb{D}}(t)}{\left|\vec{r}_{+}\right|^{2}}+\frac{\partial_{t} \vec{R}(t) \cdot \vec{r}_{+}}{\pi r_{0}^{2}} \mathfrak{m}^{(c)} .
$$

Equations (89) enable us to express the fields outside and inside the vortex in terms of the total dipole moment (84) and the velocity of the vortex, as the solutions in all space are given by Eqs. (77-79). Moreover, the symmetry of the sources in Eqs. (89) dictate that only terms with $\nu= \pm 1$ are excited (dipole approximation). Substituting Eq. (77) into Eq. (89a) we obtain the matching condition for the radial functions

$$
\theta_{ \pm 1}\left(r_{0}+0^{+}\right)-\theta_{ \pm 1}\left(r_{0}-0^{+}\right)= \pm \frac{i \mathbb{D}_{\mp}(\omega)}{2 r_{0}}
$$

where the complex dipole moment is given by

$$
\mathbb{D}_{ \pm}(\omega)=\mathbb{D}_{x}(\omega) \pm i \mathbb{D}_{y}(\omega) ;\left[\mathbb{D}_{+}(\omega)\right]^{*}=\mathbb{D}_{-}(-\omega),
$$

and $\overrightarrow{\mathbb{D}}(t)=\sum_{\omega} e^{-i \omega t} \overrightarrow{\mathbb{D}}(\omega)$. In a similar fashion Eq. (89b) yields

$$
\frac{d}{d r} \theta_{ \pm 1}\left(r_{0}+0\right)-\frac{d}{d r} \theta_{ \pm 1}\left(r_{0}-0^{+}\right)=\mp \frac{i \mathbb{D}_{\mp}(\omega)}{2 r_{0}^{2}}-\frac{i \omega R_{\mp}(\omega)}{2 \pi r_{0}^{2}} \mathfrak{m}^{(c)},
$$

with

$$
R_{ \pm}(\omega)=R_{x}(\omega) \pm i R_{y}(\omega) ;\left[R_{+}(\omega)\right]^{*}=R_{-}(-\omega),
$$

and $\vec{R}(t)=\sum_{\omega} e^{-i \omega t} \vec{R}(\omega)$.

We can now complete our solution of the radiation problem. We use solutions (78b) and (79) for $m \pm 1$, and the asymptotics of the Hankel function

$$
\frac{i \pi}{2} H_{1+\eta}^{(1)}(x)=\frac{1}{x}\left(\frac{2}{x}\right)^{\eta}+\frac{i \pi}{4} x\left(\frac{x}{2}\right)^{\eta}
$$

for $x, \eta \ll 1$. (The second term in this expansion is imaginary and therefore it should be kept although it is small in comparison with first large - but real term). As $\omega r_{0} \ll 1$, Eqs. (89) can be solved by iterations. Neglecting first the field inside the vortex core $r<r_{0}$, we obtain from Eq. (90c) the field in the exterior domain:

$$
\theta_{ \pm 1}\left(r>r_{0}\right)=\frac{\pi \omega}{4}\left(\frac{\omega r_{0}}{2}\right)^{ \pm \omega} H_{1 \mp \omega}^{(1)}(\omega r)\left[\mp \mathbb{D}_{\mp}(\omega)-\frac{\omega}{\pi} R_{\mp}(\omega) \mathfrak{m}^{(c)}\right],
$$

where we assumed $\omega>0$. Next, we use Eq. (90b), keeping only terms with real coefficients (as these are the only terms which can lead to decay of the vortex motion). Then, Eq. (78b) yields

$$
\theta_{ \pm 1}\left(r<r_{0}\right)=\frac{\pi \omega^{2} r}{8}\left(\frac{\omega r_{0}}{2}\right)^{ \pm 2 \omega}\left[\mp \mathbb{D}_{\mp}(\omega)-\frac{\omega}{\pi} R_{\mp}(\omega) \mathfrak{m}^{(c)}\right] .
$$


Now we can find the effective fields which enter into the equation of motion for the vortex (64). Using Eqs. (75), we obtain

$$
E_{ \pm} \equiv E_{x} \pm i E_{y}=\frac{i \pi \omega^{2}}{4}\left(\frac{\omega r_{0}}{2}\right)^{\mp 2 \omega}\left[-\mathbb{D}_{ \pm}(\omega) \pm \frac{\omega}{\pi} R_{ \pm}(\omega) \mathfrak{m}^{(c)}\right]
$$

for the electric field, and

$$
\nabla_{ \pm} B=\frac{i \pi \omega^{3}}{4}\left(\frac{\omega r_{0}}{2}\right)^{\mp 2 \omega}\left[ \pm \mathbb{D}_{ \pm}(\omega)-\frac{\omega}{\pi} R_{ \pm}(\omega) \mathfrak{m}^{(c)}\right] ; \quad \nabla_{ \pm} \equiv \nabla_{x} \mp i \nabla_{y}
$$

for the density.

Equations (93) can be further simplified with the help of the equations of motion of the vortex (64) and the expression of for total dipole moment (84). Differentiating Eq. (84) over time, expressing $\partial_{t} \vec{R}$ in terms of the force (51), and using Eq. (64) we find

$$
\partial_{t} \overrightarrow{\mathbb{D}}=\hat{\varepsilon} \vec{E}+\frac{\hat{\varepsilon} \vec{\nabla} B}{2 \pi} \mathfrak{m}^{(c)}
$$

Substituting Eq. (94) and $\partial_{t} \vec{R}=\hat{\varepsilon} \vec{E}-\hat{\varepsilon} \vec{f}$, keeping only leading in $\omega \ll 1$ terms, and allowing for negative $\omega$ we obtain simple expressions

$$
\begin{aligned}
& E_{ \pm}=\frac{-i \pi f_{ \pm}(\omega) \omega|\omega|}{4 \pi}\left(\frac{|\omega| r_{0}}{2}\right)^{\mp 2 \omega} \mathfrak{m}^{(c)} ; \quad f_{ \pm}=f_{x} \pm i f_{y} \\
& \nabla_{ \pm} B=\frac{\mp i \pi f_{ \pm} \omega^{2}|\omega|}{4 \pi}\left(\frac{|\omega| r_{0}}{2}\right)^{\mp 2 \omega} \mathfrak{m}^{(c)}
\end{aligned}
$$

It is worth emphasizing that the dipole moment $\mathbb{D}$ has disappeared from the equations for the field. This is because according to equation of motion of the decoupled vortex (70), the dipole moment due to the motion of the vortex itself is compensated by the dipole moment of the cut, as illustrated in Fig. 6 .

Finally, we are ready to solve the equation of motion (64) taking into account the self-consistent field created by the vortex motion. Looking for solution in a form similar to Eq. (71)

$$
f_{+} \equiv f_{x}+i f_{y}=F e^{i \Omega^{*} t} ; \quad f_{-} \equiv f_{x}-i f_{y}=F^{*} e^{-i \Omega t},
$$

we obtain from Eq. (64)

$$
\left[\frac{\Omega}{\Omega_{c}(|F|)}-1\right] f_{-}=\frac{\nabla_{-} B}{\pi} \mathfrak{m}^{(c)},
$$

where the frequency of the oscillation of the vortex decoupled from the phonons, $\Omega_{c}(|F|)$, is given by Eq. (72). Substituting the second of Eqs. (95) into Eq. (97), we obtain

$$
\Omega(|F|)=\Omega_{c}(|F|)+\frac{i}{2 \tau[\Omega(|F|)]} ; \quad \frac{1}{\tau(\omega)}=\frac{\omega^{4}}{2 \pi}\left[\mathfrak{m}^{(c)}\right]^{2}
$$




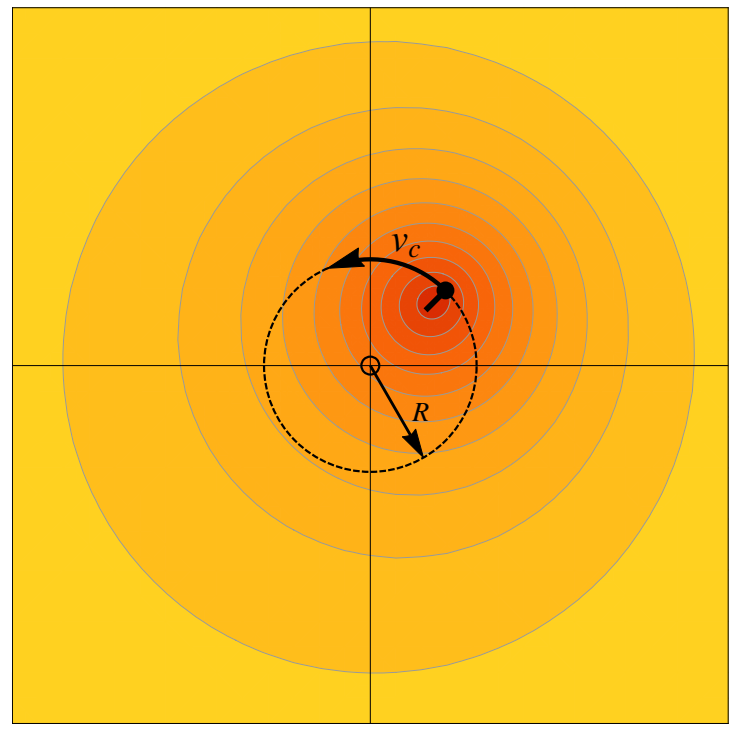

Figure 6: Rotating vortex (black dot) and its dipole moment (cut). At the distances larger than the healing length the density restores its form for a motionless vortex at $R=0$.

where we neglect the factor $\left(\omega r_{0} / 2\right)^{2 \omega} \simeq 1$ in the expression for the decay rate $1 / \tau$.

The frequency dependence of the relaxation rate, $\tau_{c}^{-1} \propto \omega^{4}$, deserves some discussion. Were the radiation simply that of a dipole $\propto R$ in two-dimensional electrodynamics, one would obtain radiation power $\simeq R^{2}\left|\omega^{3}\right|$. As the kinetic energy (in our units) is proportional to $\omega^{2} R^{2} / \Omega_{c}$, it would imply $1 / \tau \propto \Omega_{c}^{2}$. However, as we mentioned above (see also Fig. 6), the effective dipole moment vanishes and the only reason for the remaining coupling is the time dependence due to the motion of the magnetic moment $\mathfrak{m}^{(c)}$. Now, as is well known from classical electrodynamics, the power of magnetic moment radiation is smaller than that of electric dipole radiation by an extra second power of the frequency (in the low frequency limit). This is the reason for the $\Omega_{c}^{4}$ dependence of the relaxation rate.

A last point we should discuss is the value of the core magnetic moment $\mathfrak{m}^{(c)}$. At $r_{0} \gg 1$, the energy of the vortex currents that accumulated up to a distance $r_{0}$ is $\Delta E \approx \pi B \ln \left(r_{0} \sqrt{B}\right)$. This would imply that the value of the core magnetic moment is scale dependent

$$
\mathfrak{m}^{(c)}=\pi \ln r_{0} ;
$$

Getting rid of the scale dependence requires consideration of the current near the vortex with higher accuracy [perturbative treatment of the last term in Eq. (75a) and the right-hand-sided in Eq. (76a)]. Such a treatment leads to a replacement of $\ln r_{0} \rightarrow \ln \left(1 / \Omega_{c}\right) \simeq \ln \ln (1 /|F|)$ in this estimate. We do not 
believe that this double logarithmic dependence is relevant or observable and pursue this issue no further.

6.3.3. Elastic scattering of phonons by an oscillating vortex.

To study the scattering problem one should consider the field outside the effective vortex core to be in the form of a cylindrical wave, see Eq. (80). As we already saw, for the weakly oscillating vortex only the dipole mode, $\nu= \pm 1$, contributes, and in what follows we shall focus our attention on the $\nu=1$ mode:

$$
\theta_{1, \omega}(r)=A_{1, \omega}\left[J_{1-\omega}(\omega r) \cos \tilde{\gamma}_{1}-Y_{1-\omega}(\omega r) \sin \tilde{\gamma}_{1}\right],
$$

where the wave amplitude $A_{1, \omega}$ is assumed to be sufficiently small so that all the equations can be linearized with respect to this amplitude.

Let us neglect the decay of the free vortex motion due to the radiation and study the effect of the additional wave field (100) on the vortex motion:

$$
\left(\begin{array}{l}
f_{-} \\
R_{-} \\
\mathbb{D}_{-}
\end{array}\right)=\left(\begin{array}{l}
F^{*} \\
R^{*} \\
\mathbb{D}^{*}
\end{array}\right) e^{-i \Omega_{c} t}+\left(\begin{array}{l}
\delta f_{-} \\
\delta R_{-} \\
\delta \mathbb{D}_{-}
\end{array}\right) e^{-i \omega t}
$$

where all the coefficients $\delta$. are linear in $A_{1, \omega}$.

Linearizing Eq. (64) with respect to the small perturbation $\delta$. yields

$$
\left[\frac{\omega}{\Omega_{s}(|F|)}-1\right] \delta f_{-}=\frac{\nabla_{-} \delta B}{\pi} \mathfrak{m}^{(c)}
$$

where $\nabla_{-}$is defined in Eq. (93b). The eigen-frequency for this small oscillation $\Omega_{s}(|F|)$ is different from the effective cyclotron frequency $\Omega_{c}(|F|)$ due to the logarithmic dependence of the frequency on the period of the oscillations:

$$
\frac{1}{\Omega_{s}(|F|)}=\frac{1}{\Omega_{c}(|F|)}-\frac{1}{2}
$$

In analogy with our solution of the radiation problem, we utilize Eqs. (90) and the asymptotics of the Bessel and Neumann functions

$$
Y_{1+\eta}^{(1)}(x)=\frac{x}{2}\left(\frac{x}{2}\right)^{\eta} ; \quad Y_{1+\eta}^{(1)}(x)=-\frac{2}{\pi x}\left(\frac{2}{x}\right)^{\eta}
$$

for $x, \eta \ll 1$.

Instead of Eq. (91), we obtain

$$
A_{1, \omega} \sin \tilde{\gamma}_{1}=\frac{i \pi \omega}{4}\left(\frac{2}{\omega r_{0}}\right)^{\omega}\left[\delta \mathbb{D}_{-}+\frac{\omega}{\pi} \delta R_{-} \mathfrak{m}^{(c)}\right],
$$

and then Eq. (92) gives

$$
\theta_{1}\left(r<r_{0}\right)=\frac{r \omega}{2} A_{1, \omega} \cos \tilde{\gamma}_{1}\left(\frac{2}{\omega r_{0}}\right)^{\omega} .
$$


Equation (105) immediately produces,

$$
\delta E_{-}=-i \omega A_{1, \omega} \cos \tilde{\gamma}_{1}\left(\frac{2}{\omega r_{0}}\right)^{\omega} ; \quad \nabla_{-} \delta B=i \omega^{2} A_{1, \omega} \cos \tilde{\gamma}_{1}\left(\frac{2}{\omega r_{0}}\right)^{\omega}
$$

Repeating the same arguments as those leading to Eqs. (95), we find

$$
A_{1, \omega} \sin \tilde{\gamma}_{1}=\frac{i \pi \omega \delta f_{-}}{4 \pi}\left(\frac{2}{\omega r_{0}}\right)^{\omega} \mathfrak{m}^{(c)}
$$

Substituting Eqs. (106) and (107) into the linearized vortex equation of motion (102) we obtain

$$
\left[\frac{\omega}{\Omega_{s}(|F|)}-1\right] \delta f_{-}=-\frac{\delta f_{-}}{2 \omega \tau(\omega)} \cot \tilde{\gamma}_{1}(\omega)
$$

where the decay rate $1 / \tau(\omega)$ is given by Eq. (98).

Finally, using Eq. (81), we obtain the complete expression for the scatterring phase:

$$
\gamma_{1}(\omega)=\frac{\pi \omega}{2}+\arctan \left(\frac{\Omega_{s}(|F|)}{2\left[\Omega_{s}(|F|)-\omega\right] \omega \tau(\omega)}\right)
$$

where we have chosen $0<\arctan (x)<\pi$. The overall phase dependence is shown in Fig. 7.

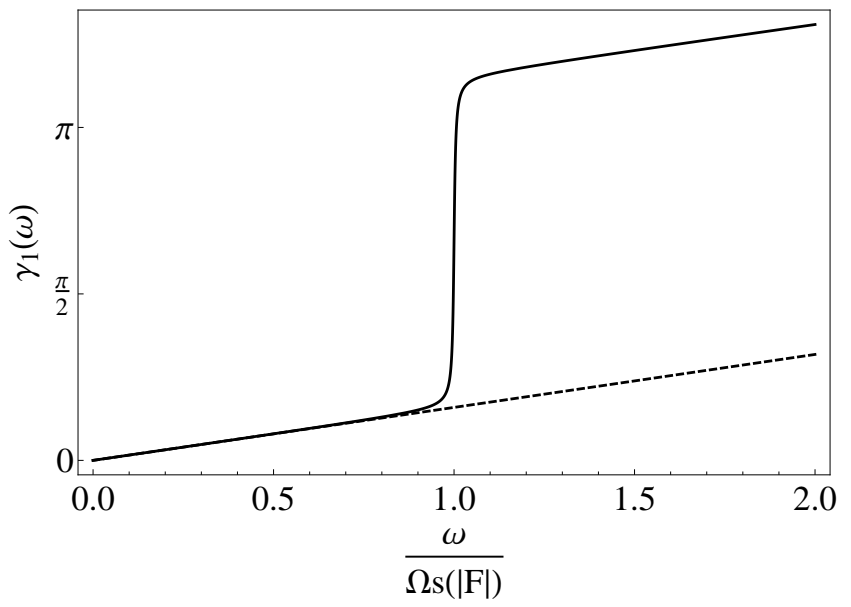

Figure 7: The elastic scattering phase in $\nu=1$ channel as the function of frequency of the incoming wave. The dashed line is the Aharonov-Bohm phase [first term in Eq. (108)]. The narrow jump corresponds to the resonance scattering by the oscillation of the vortex.

The first term in the right hand side of the above equation comes from scattering on Aharonov-Bohm flux created by the vortex. This has been discussed extensively in the literature, see e.g. Ref. [30]. The second term is due to the 
excitation of the vortex into circular motion by the incident waves and, then, re-emission of this excitation. Far from resonance this contribution is negligible, however, when $\omega \rightarrow \Omega_{s}(|F|)$, it produces unitary scattering in the $\nu=1$ channel. This resonance is narrow, and, therefore, its contribution is most likely irrelevant for the scattering of phonons with a broad quasiequilibrium distribution. However, when the system is out of equilibrium, the scattering of phonons on the vortices produces a distribution function with a singularity at the frequency of the vortex motion. These non-equilibrium phonons are strongly scattered on other vortices, providing a kinetic mechanism for vortex-vortex interaction, as the relaxation rate of the phonon energy is extremely long. We hope to come back to a detailed study of this mechanism in the nearest future.

\section{Semiclassical quantization of the vortex motion.}

In the previous section we established that the cyclotron motion of the excited vortex is a long living excitation in the classical sense, see Eq. (98), i.e. the classical motion is almost single-periodic. In quantum mechanics a singleperiodic motion corresponds to discrete excitation levels, and the classical decay rate becomes the characteristic broadening of those levels.

To calculate the position of the discrete levels $\mathcal{E}_{j}^{V}$, it is convenient to use the quantum-to-classical correspondence principle (differential form of the BohrSommerfeld quantization condition)

$$
\mathcal{E}_{j+1}^{V}-\mathcal{E}_{j}^{V}=\hbar \Omega_{c}\left(\mathcal{E}_{j}^{V}\right)
$$

where $\Omega_{c}\left(\mathcal{E}_{j}^{V}\right)$ is the frequency of the periodic motion at energy $\mathcal{E}_{j}^{V}$. For harmonic oscillators, the frequency $\Omega_{c}\left(\mathcal{E}_{j}^{V}\right)$ is independent of energy and Eq. (109) gives the exact spectrum. In our case, the frequency of the periodic motion $\Omega_{c}$ from Eq. (72) is amplitude dependent and we need to express $|\vec{f}|$ in terms of the energy of the system.

To accomplish this task, we consider the effective action (50) - (52) for one vortex. Fixing $\vec{E}=0, B=1$ inside the vortex, and restoring the physical units as described after Eqs. (1), we find the effective action of the vortex:

$$
\mathcal{S}_{V}=\int d t \mathcal{L}_{V}
$$

with the Lagrangian

$$
\mathcal{L}_{V}=\frac{\left(n_{0} m \xi^{2}\right) \pi}{\alpha^{2}}\left(\partial_{t} \vec{R}\right)^{2} \ln \frac{\hbar}{m \xi\left|\partial_{t} \vec{R}\right|}-2 \pi \vec{a}(\vec{R}(t)) \cdot \partial_{t} \vec{R}(t),
$$

where $\vec{\nabla} \times \vec{a}=\hbar n_{0}$. Thus the energy of the vortex, $\mathcal{E}^{V}$, is given by

$$
\mathcal{E}^{V}=\partial_{t} \vec{R} \cdot \frac{\partial \mathcal{L}_{V}}{\partial\left(\partial_{t} \vec{R}\right)}-\mathcal{L}_{V}=\frac{\left(n_{0} m \xi^{2}\right) \pi}{\alpha^{2}}\left(\partial_{t} \vec{R}\right)^{2}\left[\ln \frac{\hbar}{m \xi\left|\partial_{t} \vec{R}\right|}-1\right]
$$


The cyclotron frequency (72) in the original units takes the form,

$$
\Omega_{c}=\frac{\hbar \alpha^{2}}{m \xi^{2}\left[\ln \frac{\hbar}{m \xi\left|\partial_{t} \vec{R}\right|}-\frac{1}{2}\right]}
$$

Thus for circular motion, $\left(\partial_{t} \vec{R}\right)^{2}=\Omega(E)^{2} R^{2}(E)$, we obtain, with logarithmic accuracy,

$$
R^{2}\left(\mathcal{E}_{j+1}^{V}\right)-R^{2}\left(\mathcal{E}_{j}^{V}\right)=\frac{1}{n_{0} \pi}
$$

with the solution

$$
R^{2}\left(\mathcal{E}_{j}^{V}\right)=\frac{j+1 / 2}{n_{0} \pi}
$$

(The additive term $1 / 2$ is exact for harmonic oscillator and should not be that different for our problem as the non-linearity is only logarithmical). We see that the amplitude of the quantum mechanical oscillation of the vortex position for the ground state of the vortex, $j=0$, is precisely the interparticle distance.

Finally, substituting Eq. (115) into Eq. (112) we obtain with logarithmic accuracy the discrete levels

$$
\mathcal{E}_{j}^{V}=\frac{\hbar^{2} \alpha^{2}}{m \xi^{2}}\left[\frac{2 j+1}{\ln \frac{\pi n_{0} \xi^{2}}{\alpha^{4}(j+1 / 2)}}\right] .
$$

(The energy of zero-point motion $j=0$ can not be separated form the much larger mean-field core energy). For typical Bose condensates made of Rb atoms the healing length is of order of a micron and the effective two dimensional density is typically $5 \cdot 10^{9} \mathrm{~cm}^{-2}$, see e.g. Ref. [17]). Thus the excitation energy of the vortex is of order of $40 \mathrm{~Hz}$.

Equation (116) is the main result of this section. It gives a complete description of the quantized energy levels of the vortex motion. We emphasize that the condition of weak interaction, $n_{0} \xi^{2}=1 / \lambda \gg 1$, must be fulfilled in order for the energy levels (116) to be within the applicability range of the theory. In the limit of strong interaction all these levels are not relevant for the low energy spectrum.

Finally, we address the question of inter-level transitions due to spontaneous phonon-emission. The rate of this transition can be extracted directly from the classical relaxation rate (98) by the requirement that the energy loss calculated quantum-mechanically coincide with the classical calculation. Restoring the units defined after Eqs. (1), we find

$$
\frac{1}{\tau_{(j+1) \rightarrow j}}=\left(\frac{\mathcal{E}_{j}^{V}}{2 \pi \hbar}\right)\left(\frac{m \xi^{2}\left(\mathcal{E}_{j+1}^{V}-\mathcal{E}_{j}^{V}\right)}{\hbar^{2}}\right)^{3}\left[\mathfrak{m}^{(c)}\right]^{2}
$$

The absorption rate and rate of the induced emission can be found from Eq. (117) using the usual Einstein relation and the equation for the kinetics of level occupation can be obtained. 


\section{Conclusions and outlook.}

In this paper we studied the deformation of the internal structure of the vortex in two-dimensional superfluid due to a force, $\vec{f}$, acting on the vortex. Contrary to the conventional expectation that such deformation is small and analytic in $\vec{f}$, we showed in Sec. 4 that it is, in fact, non-analytic, and results in an anomalous dipole moment of the vortex $\propto \vec{f} \ln (1 /|\vec{f}|)$. We incorporated the effects of this dipole moment into the effective theory of superfluid defined on distances larger than the healing length, see Sec. 5. Armed with this effective theory, we investigated the dynamics of the vortex and its coupling to the phonons, see Sec. 6. We found that the oscillatory motion of the vortex is characterized by low frequency, see Eq. (72), which depends logarithmically on the amplitude of the oscillations and increases alongside it. We also found that the oscillations of the vortex have a long classical lifetime, see Eq. (98), and thus can be quantized, see Sec. 7 .

The lower bound on the oscillation frequency of the mode is determined by the ratio of the amplitude of the quantum motion of the vortex (which is of the order of the inter-particle distance) to healing length. In the strong coupling region, such as in ${ }^{4} \mathrm{He}$ superfluid, the healing length and the interparticle distance are of the same order and the excitations considered in this paper are irrelevant since they are of high-energy and short lifetime. On the other hand, for the weakly interacting system (which may be realized, e.g., using cold atoms) the healing length is much larger than the inter-particle distance, and the vortex excitations are well within the phonon spectrum. In this limit, the energy levels of the excited vortex may affect the phonon kinetics, because the energy dependence of the elastic phonon scattering on the vortices acquires resonance features, see Sec. 6.3.3.

As a parting statement, we enumerate some possible further developments on effects of the non-analytic core deformation.

1. Internal structure of vortex-antivortex pairs. In this paper we considered only vortices separated by distances much larger than the healing length, and therefore the deformation of their cores could be considered independently. However, when vortices come closer together the situation becomes more complicated. To illustrate, if one considers an vortex-antivortex pair (separated by a distance $R$ ) at rest, each of them will experience a Magnus force due to the superfluid flow created by the other vortex. As a result, each vortex will produce a cut directed at it pair. As the intervortex distance goes down, the Magnus force grows and so does the size of the cuts. Our preliminary study [26] indicates that at $R \lesssim 10$ the solution of the two cuts becomes less favorable than a "string" solution (a line of zero density connecting the vortex and antivortex). The quantum tunnelling between those two stable solutions ("quantum string breaking") as well as further annihilation of the vortex-antivortex pair should be important for the study of vortex kinetics and of the effects of vortex-antivortex dipoles on the phonon spectra. 
2. Strong non-linearity for vortices driven by monochromatic radiation. If we neglect quantum effects, the frequency of oscillation $\Omega_{c}$ is a an increasing function of the oscillation amplitude $F$, see Eq. (72). Consider, now, the application of monochromatic radiation with amplitude $A$ and the frequency $\omega$, on the vortex. It is known from the classical mechanics (Duffing oscillator) that such a system contains solutions corresponding to small oscillations $F \propto A$, but also solutions determined by the condition $\Omega(F) \simeq \omega$, see e.g. [31]. This latter solution turns out to be stable. Thus, in the absence of quantum effects arbitrarily small forces lead to a finite amplitude of the vortex oscillations. Quantum mechanics (see Sec. 7) puts a lower bound on frequency $\omega>\Omega_{1}-\Omega_{0}$, and on the amplitude of the force. If those restriction are fulfilled the non-linearity of the vortex dynamics still takes place, and reveals itself in strongly non-equlibrium peaked distribution function over energy levels of the vortex. The kinetics of such processes deserves further study.

3. Generalization to three dimensional systems and higher spin bosons. Our analysis was deliberately limited to the case of spinless bosons in two spatial dimensions where the description of the vortex singularities was somewhat trivial [vortex line in $2+1$ dimensional space]. The obvious generalizations of Popov's formalism are to space of higher dimension [vortex is a surface in $3+1$ dimensional space] and to bosons with higher spin [vortex is characterized not only by its position but also by the direction in the spin sector[32]]. Even though the precise mathematical tools should be developed we believe that the requirement of the non-anlytic reconstruction of the vortex core is robust and the slow oscillatory motion of the vortex should reveal itself for such systems as well.

\section{Acknowledgement}

We are grateful to $\mathrm{V}$. Cheianov for valuable discussions on the initial stage of this work and to R. Fattal and D. Klein for help and advice on the numerical work in this paper. We also thank A. Abanov, Y. Galperin, N. Prokofiev, and L. Radzihovsky for reading the manuscript and remarks, and to L. Glazman and A. Kitaev for a discussion of the results. This research has been supported by the United States-Israel Binational Science Foundation (BSF) grant No. 2012134 (O.A. and A.K.) and Simons foundation (O.A. and I.A.).

\section{Appendix A. The numerical procedure}

In Sec. 4.1 we gave an overview of our solution of Popov's equations. This consisted of solving the fictitious-time dependent equations, (30) and (28a). In what follows, we give a step-by-step description of the algorithm. 
The discrete form of the equations that we solve are:

$$
\begin{aligned}
D_{x} e_{x}^{k+1, i-1 / 2, j}+D_{y} e_{y}^{k+1, i, j-1 / 2} & =-\frac{2 \pi}{\Delta^{2}} \delta_{i, R_{v x}} \delta_{j, R_{v y}} \\
D_{t} \rho^{k, i, j} & =\mathcal{H}^{k, i, j} \rho^{k+1, i, j} .
\end{aligned}
$$

Here, $f^{t, x, y}$ denotes the value of $f$ at time $t$ and positions $x, y$ on the lattice. $\delta_{i, j}$ is the Kronecker delta. The various difference operators are defined by

$$
\begin{aligned}
& D_{x} f^{k, i, j}=\Delta^{-1}\left(f^{k, i+1, j}-f^{k, i, j}\right), \\
& D_{y} f^{k, i, j}=\Delta^{-1}\left(f^{k, i, j+1}-f^{k, i, j}\right), \\
& D_{t} f^{k, i, j}=\Delta_{t}^{-1}\left(f^{k+1, i, j}-f^{k+1, i, j}\right),
\end{aligned}
$$

where $\Delta, \Delta_{t}$ are, respectively, the lattice constant and time step. The electric field density $\vec{e}=\vec{E} / B$ is given by

$$
\begin{aligned}
& e_{x}^{k, i+1 / 2, j}=\frac{D_{x} a_{0}^{k, i, j}}{\left(\rho^{k, i+1 / 2, j}\right)^{2}}, \\
& e_{y}^{k, i, j+1 / 2}=\frac{D_{x} a_{0}^{k, i, j}}{\left(\rho^{k, i, j+1 / 2}\right)^{2}},
\end{aligned}
$$

and $\mathcal{H}$ is given by:

$$
\mathcal{H}^{k, i, j}=\frac{1}{2} \Delta_{L}-\frac{1}{2}\left[\left(e_{x}^{k, i, j}\right)^{2}+\left(e_{y}^{k, i, j}\right)^{2}\right]+1-\left(\rho^{k, i, j}\right)^{2}
$$

with $\Delta_{L}$ a discrete Laplace operator,

$$
\Delta_{L} f^{k, i, j}=\Delta^{-2}\left(f^{k, i+1, j}+f^{k, i-1, j}+f^{k, i, j-1}+f^{k, i, j-1}-4 f^{k, i, j}\right) .
$$

In these definitions, difference operators in space shift their operand fields by a half-lattice-constant, creating a staggered grid. Applying another difference operator returns the operands to the integer lattice. An object defined on one lattice is interpolated to the shifted lattice by averaging.

Each of Eqs. (A.1) and (A.2) is a linear system of equations for one of the fields. Solving Eq. (A.1) yields the unknown field $a_{0}^{k+1, i, j}$, and solving Eq. (A.2) yields $\rho^{k+1, i, j}$. Each of these systems depends on the data at the previous timestep. For completeness, we write down the full equations. Eq. (A.1) reads:

$$
\begin{array}{r}
\frac{1}{\Delta}\left[\frac{\frac{1}{\Delta}\left(a_{0}^{k+1, i+1, j}-a_{0}^{k+1, i, j}\right)}{n^{k, i+1 / 2, j}}-\frac{\frac{1}{\Delta}\left(a_{0}^{k+1, i, j}-a_{0}^{k+1, i-1, j}\right)}{n^{k, i-1 / 2, j}}\right]+[i \leftrightarrow j] \\
=-\frac{2 \pi}{\Delta^{2}} \delta_{i, R_{v x}} \delta_{j, R_{v y}}
\end{array}
$$


where the $i \leftrightarrow j$ symbol implies that we switch the function of the $i, j$ - i.e. we difference in $j$ and not in $i$. The averaged density is given by:

$$
\begin{aligned}
& n^{k, i+1 / 2, j}=\left(\frac{\rho^{k, i+1, j}+\rho^{k, i, j}}{2}\right)^{2} \\
& n^{k, i, j+1 / 2}=\left(\frac{\rho^{k, i, j+1}+\rho^{k, i, j}}{2}\right)^{2}
\end{aligned}
$$

Equation (A.2) reads:

$$
\begin{aligned}
& \frac{1}{2 \Delta^{2}}\left(\rho^{k+1, i+1, j}+\rho^{k+1, i-1, j}+\rho^{k+1, i, j+1}+\rho^{k+1, i, j-1}-4 \rho^{k+1, i, j}\right) \\
& +\left[1-\left(\rho^{k, i, j}\right)^{2}\right] \rho^{k+1, i, j}-\frac{1}{2}\left[\left(e_{x}^{2}\right)^{k, i, j}+\left(e_{y}^{2}\right)^{k, i, j}\right] \rho^{k+1, i, j} \\
& \quad=\frac{1}{\Delta_{t}}\left(\rho^{k+1, i, j}-\rho^{k, i, j}\right)
\end{aligned}
$$

where

$$
\begin{aligned}
e_{x}^{k, i+1 / 2, j} & =\frac{\frac{1}{\Delta}\left(a_{0}^{k+1, i+1, j}-a_{0}^{k+1, i+1, j}\right)}{n^{k+1, i+1 / 2, j}}, \\
e_{y}^{k, i, j+1 / 2} & =\frac{\frac{1}{\Delta}\left(a_{0}^{k+1, i, j+1}-a_{0}^{k+1, i, j}\right)}{n^{k+1, i, j+1 / 2}},
\end{aligned}
$$

and

$$
\begin{aligned}
\left(e_{x}^{2}\right)^{k, i, j} & =\frac{\left(e_{x}^{k, i+1 / 2, j}\right)^{2}+\left(e_{x}^{k, i-1 / 2, j}\right)^{2}}{2}, \\
\left(e_{y}^{2}\right)^{k, i, j} & =\frac{\left(e_{y}^{k, i, j+1 / 2}\right)^{2}+\left(e_{y}^{k, i, j-1 / 2}\right)^{2}}{2} .
\end{aligned}
$$

Note that we have used $a_{0}^{k+1, \ldots}$ in Eq. (A.7a). The reason for this (as we detail in a moment), is that in practice we solve for $\rho^{k+1, i, j}$ before solving for $a_{0}^{k+1, i, j}$ and so we can already use the data at time $k+1$ when solving for $a_{0}$ in Eqs. (A.4)

We initiate our simulation by choosing some initial conditions at $k=0$. Usually we chose $\rho^{0, i, j}=1$, or alternatively started from some precalculated solution (see later on for some details), and solved eq. (A.4) once to get $a_{0}^{0, i, j}$. Then we repeat the following procedure step by step:

1. Solve eq. (A.6) to get $\rho^{k+1, i, j}$;

2. Using the just found value of $\rho$, solve eq. (A.4) to get $a_{0}^{k+1, i, j}$;

3. $k \rightarrow k+1$;

4. Repeat until $\left|\frac{\mathcal{S}_{P}^{k+1}-\mathcal{S}_{P}^{k}}{\mathcal{S}_{P}^{k+1}}\right|<\varepsilon$; 
In the last line, $\mathcal{S}_{P}^{k}$ is the numerically computed free-energy (19) and $\varepsilon \ll 1$ is some stopping parameter.

As mentioned in the body of the text, we used MATLAB ${ }^{\mathrm{TM}}$ 's stabilizedbiconjugate-gradients method for the actual solving of the linear systems. This choice was one of convenience and we don't believe that it is necessarily better than other accepted sparse system solvers such as SOR. We also used preconditioning to enhance convergence. We used MATLAB ${ }^{\mathrm{TM}}$ 's incomplete LU decomposition to generate preconditioning matrices for the operators in eqs. $($ A.3g) $+($ A.7a). We omit further details of these minor points for brevity's sake.

We found out, by trial and error, that we could get much better convergence and stability if instead of starting from the high energy initial condition $\rho=1$, we started from a partially relaxed system. Our usual choice was to solve eqs. (A.4) and (A.6) as described, however with the following change: we replaced $e_{x}^{2}, e_{y}^{2}$ in Eq. (A.6) with the analytic solution for an unperturbed vortex, i.e. $e_{x}^{2}+e_{y}^{2}=1 / r^{2}$, with $r$ the radial distance. This proved so helpful that we practically hardwired it into our code, so most (possibly all) of the data in this work was obtained by this procedure.

The central result of these numerical simulations was the appearance and scaling of the cut. This behaviour is shown in Figs. 1, 4 and A.8. As a last point, we note that the cutoff point of fig. A.8 is not accidental. Rather, at higher flows a new phenomenon appears. This is the the vortex-antivortex collapse mentioned in our concluding remarks in the body of the paper.

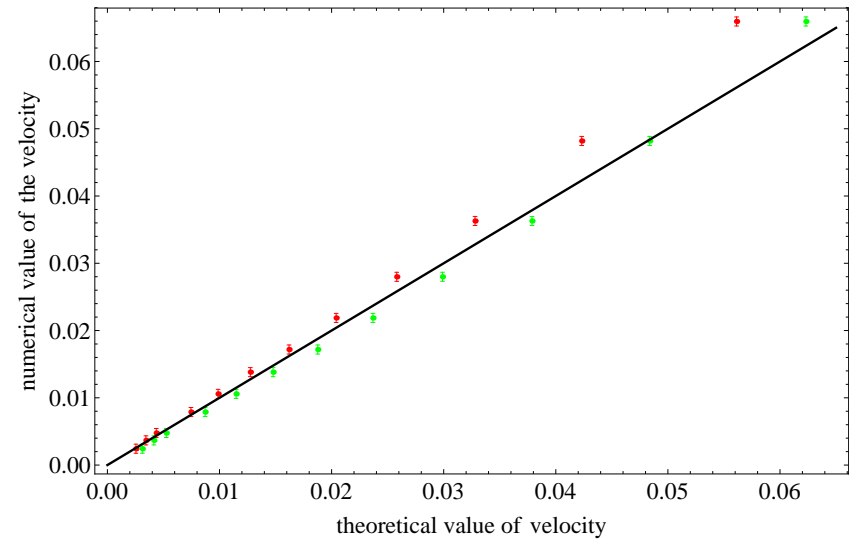

Figure A.8: The numerical value of the velocity, extracted from the cut's length, versus the actual velocity at the point of the vortex. The green and the red marks are the velocities calculated with and without the logarithmic shift of virtual position of the vortex, respectively. The error bars are due to the finite size of lattice-spacing which limits the accuracy of the measured cut's length (Additional error which comes form the finite size of the system is not included). 


\section{References}

[1] S.J. Putterman, Superfluid hydrodynamics (North-Holland series in low temperature physics), North-Holland, 1974.

[2] E.B. Sonin, Transverse force on a vortex and vortex mass: Effects of free bulk and vortex-core bound quasiparticles Phys. Rev. B, 87, 134515 (2013).

[3] N. Henkel, R. Nath, and T. Pohl, Three-Dimensional Roton Excitations and Supersolid Formation in Rydberg-Excited Bose-Einstein Condensates, Phys. Rev. Lett. 104, 195302 (2010).

[4] F. Arrigoni, E. Vitali, D.E.Galli, and L. Reatto, Excitation spectrum in two-dimensional superfluid ${ }^{4} \mathrm{He}$, Low Temp. Phys. 39, 793 (2013).

[5] , M.H. Anderson, J.R. Ensher, M.R. Matthews, C.E. Wieman, and E.A. Cornell, Observation of Bose-Einstein condensation in a dilute atomic vapor Science 269, 198 (1995).

[6] C.C. Bradley, C.A. Sackett, J.J. Tollett, R.G. Hulet, Evidence of BopeEinstein condensation in an atomic gas with attractive interactions, Phys. Rev. Lett. 75, 1687 (1995).

[7] K.B. Davis, M.O. Mewes, M.R. Andrews, N.J. Vandruten, D.S. Durfee, D.M. Kurn and W. Ketterle,Bose-Einstein condensation in gas of sodium atoms, Phys. Rev. Lett. 75, 3969 (1995).

[8] W.F. Vinen, and J.J. Niemela, Quantum turbulence, J. Low. Temp. Phys. 128, 167 (2002).

[9] M.R. Matthews, B.P. Anderson,P.C. Haljan, D.S. Hall, C.E. Wieman, and E.A. Cornell, Vortices in a Bose-Einstein condensate, Phys. Rev. Lett. 83, 2498 (1999).

[10] K.W. Madison, F. Chevy, W. Wohlleben, and J. Dalibard, Vortex formation in a stirred Bose-Einstein condensate, Phys. Rev. Lett. 84, 806 (2000).

[11] C.N. Weiler, T.W. Neely, D.R. Scherer,A.S. Bradley, M.J. Davis, and B.P. Anderson, Spontaneous vortices in the formation of Bose-Einstein condensates, Nature 455 948-U37 (2008).

[12] D.V. Freilich, D.M. Biancho, A.M. Kaufman, T.K. Langin, and D.S. Hall, Real-Time Dynamics of Single Vortex Lines and Vortex Dipoles in a BoseEinstein Condensate, Science 329, 1182 (2010).

[13] T.W. Neely, E.C. Samson, A.S. Bradley, J.M. Davis, and B.P. Anderson, Observation of Vortex Dipoles in an Oblate Bose-Einstein Condensate, Phys. Rev. Lett. 104, 160401 (2010). 
[14] N.L. Smith, W.H. Heathcote, J.M. Krueger, and C.J. Foot, Experimental observation of the tilting mode of an array of vortices in a dilute BoseEinstein condensate Phys. Rev. Lett. 98, 080406 (2004).

[15] J.R. Abo-Shaer, C. Raman, J.M. Vogels, and W. Ketterle, Observation of vortex lattices in Bose-Einstein condensates, Science, 292,476 (2001)

[16] V. Schweikhard, I. Coddington, P. Engels, S. Tung, and E.A. Cornell, Vortex-lattice dynamics in rotating spinor Bose-Einstein condensates Phys. Rev. Lett. 93, 210403 (2004).

[17] I. Coddington, P. Engels, V. Schweikhard, and E.A. Cornell, EA Observation of Tkachenko oscillations in rapidly rotating Bose-Einstein condensates, Phys. Rev. Lett. 91, 100402 (2003).

[18] E.A.L. Henn, J.A. Seman, G. Roati, K.M.F. Magalhaes, and V.S. Bagnato, Emergence of Turbulence in an Oscillating Bose-Einstein Condensate, Phys. Rev. Lett. 103, 045301 (2009).

[19] T.W. Neely, A.S. Bradley, E.C. Samson, S.J. Rooney, E.M. Wright, K.J.H. Law, R. Carretero-Gonzalez, P.G. Kevrekidis, M.J. Davis, and B.P. Anderson, Characteristics of Two-Dimensional Quantum Turbulence in a Compressible Superfluid, Phys. Rev. Lett. 111, 235301 (2013).

[20] H. Lamb, Hydrodynamics, (Cambridge University Press 1895)

[21] V. N. Popov, Functional integrals and in quantum field theory and statistical physics (Kluwer, Boston, 1983).

[22] L.M. Milne-Thomson, Theoretical Hydrodynamics (Dover, 1996).

[23] W.F. Ames, Numerical methods for partial differential equations, Academic Press, San Diego, 3rd. edition, (1992).

[24] S. L. Adler and T. Piran, Rev. Mod. Phys., 56 140, (1984).

[25] D. J. Thouless and J. R. Anglin, Vortex mass in a superfluid at low frequencies. Phys. Rev. Lett., 99105301 (2007).

[26] A. Klein, I. Aleiner, and O. Agam (in preparation).

[27] R. P. Feynman, Application of quantum mechanics to liquid helium. In C. J. Gorter, editor, Progress in Low Temperature Physics, volume 1. NorthHolland, Amsterdam, (1955).

[28] V. N. Popov, Quantum vortices and phase transitions in Bose systems. Sov. Phys. JETP, 37 341, (1973).

[29] J. M. Duan and A. J. Leggett, Inertial mass of a moving singularity in a Fermi superuid. Phys. Rev. Lett., 681216 (1992). 
[30] E.B. Sonin, Magnus force in superfluids and superconductors, Phys. Rev. B 55, 485 (1997).

[31] M. Dykman Fluctuating nonlinear oscillators (Oxford, 2012).

[32] Y. Kawaguchi and M. Ueda, Spinor Bose-Einsten condensates, Phys. Rep. 520, 253 (2012).

[33] Y. Saad, Iterative methods for sparse linear systems, (Society for Industrial and Applied Mathematics, 2003). 